\title{
HOMOGENIZATION OF PERIODIC DIFFERENTIAL OPERATORS OF HIGH ORDER
}

\author{
N. VENIAMINOV
}

\begin{abstract}
A periodic differential operator of the form $A_{\varepsilon}=\left(\mathbf{D}^{p}\right)^{*} g(\mathbf{x} / \varepsilon) \mathbf{D}^{p}$ is considered on $L_{2}\left(\mathbb{R}^{d}\right)$; here $g(x)$ is a positive definite symmetric tensor of order $2 p$ periodic with respect to a lattice $\Gamma$. The behavior of the resolvent of the operator $A_{\varepsilon}$ as $\varepsilon \rightarrow 0$ is studied. It is shown that the resolvent $\left(A_{\varepsilon}+I\right)^{-1}$ converges in the operator norm to the resolvent of the effective operator $A^{0}$ with constant coefficients. For the norm of the difference of resolvents, an estimate of order $\varepsilon$ is obtained.
\end{abstract}

\section{$\S 1$. INTRODUCTION}

1.1. On homogenization theory. Problems related to physical processes in a crystal arise naturally in solid state physics. From the point of view of mathematical physics, these problems lead to an investigation of differential operators (DO) that are periodic with respect to a certain lattice $\Gamma$ describing the crystal structure.

An approximation in a small spacial grid period (in a certain precise meaning to be clarified later on) of operators with periodic coefficients by operators with constant coefficients is of great interest. Such a simplification of an initial operator is called homogenization or averaging. The limiting operator with constant coefficients describes an averaged homogeneous medium.

Homogenization theory is a vast area of theoretical and applied studies. A lot of valuable results were obtained and many specific methods were developed in the field. First of all, the reader is referred to $[\mathrm{BaPa}, \mathrm{BeLP}, \mathrm{ZKO}]$. This list is to no extent exhaustive and does not cover all the existing results.

1.2. Operator-theoretic approach to the homogenization theory. Over the past ten years, in the work of Birman and Suslina, a new operator approach to the averaging of periodic DOs of the second order was proposed. A significant limitation of this approach is that the initial operator $A$ is assumed to admit a factorization as the product of mutually conjugate first order differential operators:

$$
A=X^{*} X .
$$

A fundamental result of this theory is the following theorem on approximation of the resolvent in the operator norm (see [BS1]).

Theorem 1.1 (Birman, Suslina). Let $\Gamma$ be a lattice in $\mathbb{R}^{d}$, and let $g=g(\mathbf{x})$ be a $\Gamma$-periodic $(m \times m)$-matrix satisfying the relations

$$
\nu_{1}|\zeta|^{2} \leq\langle g(\mathbf{x}) \zeta, \zeta\rangle \leq \nu_{2}|\zeta|^{2}, \quad \zeta \in \mathbb{C}^{m}, \quad \mathbf{x} \in \mathbb{R}^{d},
$$

2010 Mathematics Subject Classification. Primary 35B27.

Key words and phrases. Periodic differential operators, averaging, homogenization, threshold effect, operators of high order. 
where $0<\nu_{1} \leq \nu_{2}<+\infty$ are some constants. Suppose that $m \geq n$ and $b(\mathbf{k})$ is an $(m \times n)$-matrix first degree homogeneous in $\mathbf{k} \in \mathbb{R}^{d}$ and such that $\operatorname{rank} b(\mathbf{k})=n$ for $\mathbf{k} \neq 0$. Consider the $\Gamma$-periodic second order differential operator

$$
A(g)=b(\mathbf{D})^{*} g(\mathbf{x}) b(\mathbf{D})
$$

and the related family of $(\varepsilon \Gamma)$-periodic operators $A_{\varepsilon}(g)=A(g(\cdot / \varepsilon))$. Then there exists a constant matrix $g^{0}$ such that the effective differential operator $A^{0}=A\left(g^{0}\right)$ approximates the family of the operators $A_{\varepsilon}$ in the following sense:

$$
\left\|\left(A_{\varepsilon}(g)+I\right)^{-1}-\left(A^{0}+I\right)^{-1}\right\|_{L_{2}\left(\mathbb{R}^{n}\right) \rightarrow L_{2}\left(\mathbb{R}^{n}\right)} \leq \mathcal{C}_{\times} \varepsilon, \quad 0<\varepsilon \leq 1,
$$

where $\mathcal{C}_{\times}=\mathcal{C}_{\times}\left(\Gamma, \nu_{1}, \nu_{2}, b\right)$ is a constant.

Estimate (1.2) is order sharp and the constant $\mathcal{C}_{\times}$is controlled explicitly. Such estimates are called "operator error estimates in homogenization theory".

In other publications, Birman and Suslina obtained a number of results, including more accurate approximations of the resolvent of the operator $A_{\varepsilon}(g)$ (see [BS2, BS3]), as well as operator error estimates in the homogenization of nonstationary periodic problems (see [S, BS4]).

We explain the basic ideas of the approach of Birman and Suslina. For periodic DOs, the averaging procedure can be studied as the spectral threshold effect at the edge of the spectrum of $A(g)$. An appropriate scaling reduces the problem of obtaining (1.2) to the study of the behavior of the resolvent $\left(A(g)+\varepsilon^{2} I\right)^{-1}$ for small $\varepsilon$, i.e., of the resolvent of $A(g)$ near the edge of the spectrum (the point $\lambda=0$ ). Next, in accordance with the Floquet-Bloch theory, the operator $A(g)$ is expanded into a direct integral of operators $A(\mathbf{k} ; g)$, acting in $L_{2}(\Omega)$ (where $\Omega$ is a cell of the lattice of periods) and depending on a $d$-dimensional parameter $\mathbf{k}$ (quasimomentum). The operator $A(\mathbf{k} ; g)$ is given by $b(\mathbf{D}+\mathbf{k})^{*} g(\mathbf{x}) b(\mathbf{D}+\mathbf{k})$ with periodic boundary conditions. The behavior of the resolvent $\left(A(\mathbf{k} ; g)+\varepsilon^{2} I\right)^{-1}$ can be efficiently described in terms of threshold characteristics (eigenvalues and eigenfunctions of the operator $A(\mathbf{k} ; g)$ near the edge of the spectrum). For this, the one-dimensional parameter $t=|\mathbf{k}|$ is singled out and the family of operators $A(\mathbf{k} ; g)$, which is a quadratic operator pencil with respect to $t$, is studied by the methods of analytic perturbation theory. A significant part of the construction is carried out within the framework of an abstract operator-theoretic approach in which one studies the operator pencil of the form $A(t)=X(t)^{*} X(t)$ (where $X(t)=X_{0}+t X_{1}$ ) acting in a Hilbert space $\mathfrak{H}$. It is assumed that the subspace $\mathfrak{N}=\operatorname{Ker} A(0)$ is finite-dimensional. A key point of this abstract approach is the extraction of the so-called spectral germ $S$ of the family $A(t)$ at $t=0$. The germ $S$ is a selfadjoint operator acting in a finite-dimensional subspace $\mathfrak{N}$; it is determined by the threshold characteristics of the operator. It turns out that an approximation in the operator norm for the resolvent $\left(A(t)+\varepsilon^{2} I\right)^{-1}$ can be found in terms of an operator of finite rank, namely, the resolvent of $t^{2} S$. When applied to differential operators, this abstract result leads to the desired approximation of the resolvent, because the effective DO has the same germ as the original DO.

1.3. Main result. In the present paper, we use the approach described above to investigate operators of higher order. A restriction of the form (1.1) is still crucial. We study the operator

$$
A(g)=\left(\mathbf{D}^{p}\right)^{*} g(\mathbf{x}) \mathbf{D}^{p},
$$

where $g(\mathbf{x})$ is a symmetric positive definite tensor periodic with respect to the lattice $\Gamma$, and $\mathbf{D}=-i \nabla$ is the operator of differentiation. The precise definition of the operator (1.3) is given in 92 . In particular, an operator of the form (1.3) for $p=2$ is studied in the elasticity theory of plates (see [ZKO]). For the operators $A_{\varepsilon}=A(g(\cdot / \varepsilon)$ ) of order $2 p$, 
an analog of Theorem 1.1 is obtained. Namely, the effective operator $A^{0}=\left(\mathbf{D}^{p}\right)^{*} g^{0} \mathbf{D}^{p}$ is constructed, and it is shown that the resolvent of $A_{\varepsilon}$ converges in the operator norm of $L_{2}\left(\mathbb{R}^{d}\right)$ to the resolvent of the effective operator $A^{0}$, and there is an estimate of the form (1.2) for the difference of the resolvents. This estimate is order sharp, and the constant in the estimate is controlled explicitly in terms of $d, p,\|g\|_{\infty},\left\|g^{-1}\right\|_{L_{\infty}}$, and the parameters of the lattice $\Gamma$. This is the main result of the paper (see Theorem 2.3).

1.4. Method of investigation. Our method is a further development of the approach proposed in BS1. With the help of a scaling transformation, an estimate of the form (1.2) for an operator of order $2 p$ is reduced to the inequality

$$
\varepsilon^{2 p-1}\left\|\left(A(g)+\varepsilon^{2 p} I\right)^{-1}-\left(A^{0}+\varepsilon^{2 p} I\right)^{-1}\right\|_{L_{2}\left(\mathbb{R}^{d}\right) \rightarrow L_{2}\left(\mathbb{R}^{d}\right)} \leq \mathcal{C}_{\times} \varepsilon, \quad 0<\varepsilon \leq 1 .
$$

Next, the operator $A(g)$ is expanded into a direct integral of operators $A(\mathbf{k} ; g)$ acting in $L_{2}(\Omega)$ and depending on the quasimomentum $\mathbf{k}$. The operator $A(\mathbf{k} ; g)$ is given by $\left((\mathbf{D}+\mathbf{k})^{p}\right)^{*} g(\mathbf{x})(\mathbf{D}+\mathbf{k})^{p}$ with periodic boundary conditions. As in [BS1], a one-dimensional parameter $t=|\mathbf{k}|$ is singled out; with respect to this parameter the family $A(\mathbf{k} ; g)$ is a polynomial operator pencil of degree $2 p$. An abstract operator-theoretic approach is developed in order to study it. An operator pencil of the form $A(t)=X(t)^{*} X(t)$, where $X(t)=X_{0}+t X_{1}+\cdots+t^{p} X_{p}$, is treated in abstract terms. It is assumed that the subspace $\mathfrak{N}=\operatorname{Ker} X_{0}$ is finite-dimensional and that the kernels of $X_{1}, \ldots, X_{p-1}$ contain $\mathfrak{N}$. It is possible to extract the spectral germ $S$ of the operator family $A(t)$ at $t=0$. This germ is a selfadjoint operator acting in the subspace $\mathfrak{N}$, where it is determined only by the operators $X_{0}$ and $X_{p}$ (and does not depend on the operators $\left.X_{1}, \ldots, X_{p-1}\right)$. The resolvent $\left(A(t)+\varepsilon^{2 p} I\right)^{-1}$ is approximated by a finite-rank operator, namely, by the resolvent of $t^{2 p} S$. This is the main result of the abstract part of the paper (Theorem 4.11). When applied to differential operators, this abstract result leads to the desired estimate (1.4), because the effective operator has the same spectral germ as the initial operator.

1.5. Outline of the paper. The further presentation is organized as follows. In $₫ 2$, the rigorous statement of the problem and the main result are given. In $\S \S 3$ and 4 , we develop the abstract approach to polynomial operator pencils. In \$5 the expansion of the operator $A$ into a direct integral is described and the basic properties of the DOs in question are obtained. Finally, in $₫ 6$, the abstract method described in $\S \S 3$ and 4 is applied to the DOs under study. On this basis we prove our main result (Theorem 2.3).

\section{§2. Statement of the Problem and the main Result}

2.1. Basic notation. Let $\Gamma$ be the lattice in $\mathbb{R}^{d}$ generated by the vectors $\mathbf{a}_{1}, \mathbf{a}_{2}, \ldots, \mathbf{a}_{d} \in$ $\mathbb{R}^{d}$ :

$$
\Gamma=\left\{\mathbf{a} \in \mathbb{R}^{d}: \mathbf{a}=\sum_{j=1}^{d} \nu^{j} \mathbf{a}_{j}, \quad \nu^{j} \in \mathbb{Z}\right\},
$$

and let $\Omega$ be an elementary cell of $\Gamma$ :

$$
\Omega=\left\{\mathbf{x} \in \mathbb{R}^{d}: \mathbf{x}=\sum_{j=1}^{d} \tau^{j} \mathbf{a}_{j}, \quad 0<\tau^{j}<1\right\} .
$$

The basis $\mathbf{b}^{1}, \ldots, \mathbf{b}^{d} \in \mathbb{R}^{d}$ dual to $\mathbf{a}_{1}, \ldots, \mathbf{a}_{d}$ is given by the relations $\left\langle\mathbf{b}^{i}, \mathbf{a}_{j}\right\rangle=2 \pi \delta_{j}^{i}$. This basis generates the dual lattice $\widetilde{\Gamma}$ :

$$
\widetilde{\Gamma}=\left\{\mathbf{b} \in \mathbb{R}^{d}: \mathbf{b}=\sum_{i=1}^{d} \mu_{i} \mathbf{b}^{i}, \quad \mu_{i} \in \mathbb{Z}\right\} .
$$


We denote by $\widetilde{\Omega}$ the Brillouin zone:

$$
\widetilde{\Omega}=\left\{\mathbf{k} \in \mathbb{R}^{d}:|\mathbf{k}|<|\mathbf{k}-\mathbf{b}|, 0 \neq \mathbf{b} \in \widetilde{\Gamma}\right\} .
$$

This $\widetilde{\Omega}$ is a fundamental domain for $\widetilde{\Gamma}$ : all its $\widetilde{\Gamma}$-translations are mutually disjoint and the union of their closures covers the entire $\mathbb{R}^{d}$. Also, let $r_{0}$ be the radius of a ball inscribed in $\operatorname{clos} \Omega$. Observe that

$$
2 r_{0}=\min _{0 \neq \mathbf{b} \in \widetilde{\Gamma}}|\mathbf{b}|
$$

Given a measurable $\Gamma$-periodic function $f$, we put $f^{\varepsilon}(\mathbf{x})=f\left(\varepsilon^{-1} \mathbf{x}\right)$.

For a Hilbert space $\mathfrak{H}$, we denote by $(\cdot, \cdot)_{\mathfrak{H}}$ and $\|\cdot\|_{\mathfrak{H}}$ the scalar product and the norm in $\mathfrak{H}$, respectively. If $\mathfrak{G}$ is another Hilbert space, then $\|\cdot\|_{\mathfrak{H} \rightarrow \mathfrak{G}}$ stands for the norm of a bounded operator from $\mathfrak{H}$ to $\mathfrak{G}$. If it does not lead to any misunderstanding or ambiguities, some of the indices may be dropped. In particular, if the spaces $\mathfrak{H}$ and $\mathfrak{G}$ coincide, the norm of an operator acting in $\mathfrak{H}$ will be denoted by $\|\cdot\|_{\mathfrak{H}}$. By $\langle\cdot, \cdot\rangle$ and $|\cdot|$ we designate the standard scalar product and the norm in $\mathbb{C}^{n}$.

In what follows, we write $\mathbf{x}=\left(x_{1}, \ldots, x_{d}\right) \in \mathbb{R}^{d}$,

$$
D_{j}=-i \partial_{j}=-i \frac{\partial}{\partial x_{j}}, \quad \mathbf{D}=\left(D_{1}, \ldots, D_{d}\right)=-i \nabla .
$$

If $\mathbf{b}=\left(b_{1}, \ldots, b_{d}\right) \in \mathbb{C}^{d}$ and $s \in \mathbb{N}$, we denote by $\mathbf{b}^{s} \in \mathbb{C}^{d^{s}}$ the following tensor of order $s$ :

$$
\mathbf{b}^{s}=b_{i_{1}} b_{i_{2}} \ldots b_{i_{s}}, \quad i_{j}=1, \ldots, d, \quad j=1, \ldots, s .
$$

The same notation is used for formal vectors, such as the operator $\mathbf{D}$ of differentiation. By $\left|\mathbf{b}^{s}\right|$ we denote the Euclidean norm of the tensor $\mathbf{b}^{s}$ :

$$
\left|b^{s}\right|^{2}=\sum_{1 \leq i_{1}, \ldots, i_{s} \leq d}\left|\left(\mathbf{b}^{s}\right)_{i_{1}, i_{2}, \ldots, i_{s}}\right|^{2} .
$$

Note that $\left|\mathbf{b}^{s}\right|=|\mathbf{b}|^{s}$.

Let $M$ be a normed space. The $M$-valued Sobolev space of order $s$ in a domain $G \subset \mathbb{R}^{d}$ is denoted by $H^{s}(G ; M)$ and its periodic subspace by $\widetilde{H}^{s}(G ; M)$ (for the case where $G=\Omega(\Gamma)$ is a unit cell of a lattice $\Gamma)$. For the scalar case, we drop $M=\mathbb{C}$ in the notation.

2.2. Periodic differential operators. Let $g$ be a tensor of order $2 p$ that determines a symmetric operator in a vector subspace $M \subset \mathbb{C}^{d^{p}}$. Suppose that $M$ contains all fully symmetric tensors in $\mathbb{C}^{d^{p}}$ and put $m=\operatorname{dim} M$. In a fixed basis, the symmetry property of $g$ can be expressed as invariance under the permutation of the first $p$ indices with the last $p$ indices:

$$
g_{I J}=g_{J I}^{*}, \quad I, J \in\{1, \ldots, d\}^{p} .
$$

In the last formula, $*$ means complex conjugation. Note that the subspace $M$ does not necessarily coincide with $\mathbb{C}^{d^{p}}$ in the case where the tensor $g$ possesses extra symmetries, for example, if it is invariant under any permutation of indices that does not mix the first $p$ indices with the last ones. In particular, the elasticity tensor $g$ possesses this property (see (1.3) ). For it, we have $p=2$ and

$$
g_{i j s t}=g_{s t i j}=g_{j i s t}=g_{i j t s} \in \mathbb{R}, \quad i, j, s, t \in\{1, \ldots, d\} .
$$

In this case, as $M$ we can take the subspace of all symmetric matrices, and $m=\frac{d(d+1)}{2}$.

Now, suppose that the tensor $g=g(\mathbf{x})$ is defined for all $\mathbf{x} \in \mathbb{R}^{d}, \Gamma$-periodic, and uniformly lower and upper bounded. That is, there exist constants $\nu_{1}, \nu_{2}>0$ such that

$$
\nu_{1}|\zeta|^{2} \leq\langle g(\mathbf{x}) \zeta, \zeta\rangle \leq \nu_{2}|\zeta|^{2}, \quad \zeta \in M, \quad \mathbf{x} \in \mathbb{R}^{d} .
$$


Notation 2.1. To simplify the notation, we denote the norm in the space $L_{\infty}\left(\mathbb{R}^{d}, \mathbf{B}(M)\right)$ by $\|\cdot\|_{\infty}$.

Relations (2.3) are equivalent to the fact that for each $\mathbf{x} \in \mathbb{R}^{d}$ there exists $g(\mathbf{x})^{-1}$ and

$$
g, g^{-1} \in L_{\infty}\left(\mathbb{R}^{d}, \mathbf{B}(M)\right) \text { and } g(\mathbf{x})>0, \quad \mathbf{x} \in \mathbb{R}^{d},
$$

where $\mathbf{B}(M)$ is the space of bounded operators in $M$. Relation (2.3) follows from (2.4) with

$$
\nu_{1}=\left\|g^{-1}\right\|_{\infty}^{-1}, \quad \nu_{2}=\|g\|_{\infty} .
$$

In what follows, we shall suppose that the constants $\nu_{1}, \nu_{2}$ in (2.3) are chosen optimally, as in (2.5).

Consider the $p$ th order derivative operator

$$
\mathbf{D}^{p}: L_{2}\left(\mathbb{R}^{d}\right) \rightarrow L_{2}\left(\mathbb{R}^{d} ; M\right),
$$

with the domain $\operatorname{Dom}\left(\mathbf{D}^{p}\right)=H^{p}\left(\mathbb{R}^{d}\right)$ and acting by the rule

$$
\left(\mathbf{D}^{p} u(\mathbf{x})\right)_{i_{1}, \ldots, i_{p}}=(-i)^{p} \partial_{i_{1}} \ldots \partial_{i_{p}} u(\mathbf{x}) .
$$

Remark 2.2. Since the derivatives commute, we see that $\mathbf{D}^{p} u(\mathbf{x})$ always belongs to the subspace $M$ for any x.

We introduce the following closed quadratic form defined on the same domain as the $p$ th derivative operator:

$$
\mathfrak{a}[u]=\left(g \mathbf{D}^{p} u, \mathbf{D}^{p} u\right)_{L_{2}\left(\mathbb{R}^{d}\right)} .
$$

This form gives rise to a selfadjoint operator $A$ acting in $L_{2}\left(\mathbb{R}^{d}\right)$. Formally, the operator $A$ may be written in the form

$$
A=\left(\mathbf{D}^{p}\right)^{*} g \mathbf{D}^{p}
$$

though no differentiability properties are assumed about the tensor $g$. The same notational convention will be used later in the text for other operators, where in each case the proper definition is to be understood in the sense of quadratic forms. If necessary, the dependence on the tensor coefficient will be indicated explicitly: $\mathfrak{a}=\mathfrak{a}(g), A=A(g)$.

For the operator $A(g)$, we consider a family of periodic differential operators $A_{\varepsilon}(g)=$ $A\left(g^{\varepsilon}\right)$, with rapidly oscillating coefficients as $\varepsilon \rightarrow 0$. A typical problem of homogenization theory in application to the operator $A_{\varepsilon}(g)$ is the study of the solutions of the equation

$$
A_{\varepsilon}(g) u_{\varepsilon}+u_{\varepsilon}=F, \quad F \in L_{2}\left(\mathbb{R}^{d}\right),
$$

as $\varepsilon \rightarrow 0$. Equivalently, we are interested in the behavior of the resolvent $\left(A_{\varepsilon}(g)+I\right)^{-1}$ as $\varepsilon \rightarrow 0$. Such a limiting procedure may be interpreted physically as the study of a medium with rapidly oscillating parameters. The following theorem is the main result of this paper.

Theorem 2.3. Let $g \in L_{\infty}\left(\mathbb{R}^{d}, \mathbf{B}(M)\right)$ be a $\Gamma$-periodic tensor satisfying conditions (2.1) and (2.3) uniformly in $\mathbf{x}$. Let $A$ be the operator given by (2.8). Then there exists a constant tensor $g^{0} \in \mathbf{B}(M)$ and a constant $\mathcal{C}_{\times}=\mathcal{C}_{\times}\left(d, p, \nu_{1}, \nu_{2}, \Gamma\right)$ such that

$$
\left\|\left(A_{\varepsilon}(g)+I\right)^{-1}-\left(A\left(g^{0}\right)+I\right)^{-1}\right\| \leq \mathcal{C}_{\times} \varepsilon, \quad 0<\varepsilon \leq 1 .
$$

The tensor $g^{0}$ is called the effective tensor of the problem and describes the "homogenized" medium. A constructive description can be obtained for $g^{0}$; see Subsections 6.2 and 6.3 below. The constant $\mathcal{C}_{\times}$in (2.9) can be expressed explicitly in terms of $d, p$, the constants $\nu_{1}$ and $\nu_{2}$ in (2.3), and the parameters of the lattice $\Gamma$.

For $p=1$, the results of Theorem 2.3 are a particular case of Theorem 1.1 in BS1. In the current work, Theorem 2.3 will be proved for $p \geq 2$. 


\section{§3. Abstract approach: The spectral germ}

In this section we postulate a number of properties for abstract operator families and introduce the concept of a spectral germ.

3.1. Polynomial pencils of the form $X(t)^{*} X(t)$. Let $\mathfrak{H}, \mathfrak{H}_{*}$ be separable Hilbert spaces. Consider a family of nonnegative selfadjoint operators

$$
A(t)=X(t)^{*} X(t): \mathfrak{H} \rightarrow \mathfrak{H},
$$

where the family of operators $X(t)$ is a polynomial pencil of the form

$$
X(t)=\sum_{j=0}^{p} t^{j} X_{j}, \quad t \in \mathbb{R},
$$

where $p \in \mathbb{N}$ and $p \geq 2$.

Remark 3.1. The case where $p=1$ was studied in detail in the papers [BS1, BS2, BS3]. The restriction $p \geq 2$ is introduced in the present paper for convenience, and all the results obtained generalize the known facts for $p=1$.

The operators $X(t)$ and $X_{j}$ act from the space $\mathfrak{H}$ to the space $\mathfrak{H}_{*}$,

$$
X(t), X_{j}: \mathfrak{H} \rightarrow \mathfrak{H}_{*},
$$

and are closed. It is assumed that

$$
\operatorname{Dom} X(t)=\operatorname{Dom} X_{0} \subset \operatorname{Dom} X_{j} \subset \operatorname{Dom} X_{p}=\mathfrak{H}, \quad j=1, \ldots, p-1, \quad t \in \mathbb{R} .
$$

Note that, by the closed graph theorem, $X_{p}$ is bounded. Next, we formulate the conditions imposed on the operators $X_{j}$.

Condition 3.2. Denote by

$$
\mathfrak{N}:=\operatorname{Ker} A_{0}=\operatorname{Ker} X_{0}
$$

the kernel of the operator $A_{0}=A(0)$. We assume that

$$
n:=\operatorname{dim} \mathfrak{N}<\infty,
$$

and that the point $\lambda=0$ is an isolated point of the spectrum of $A_{0}$.

Notation 3.3. By $d^{0}$ we denote the distance from zero to the rest of the spectrum of $A_{0}$. By the above condition, we have $d^{0}>0$.

Along with the family of operators $A(t)$, we shall also consider the following family, selfadjoint in $\mathfrak{H}_{*}$ :

$$
A_{*}(t):=X(t) X(t)^{*} .
$$

We denote

$$
A_{* 0}:=X_{0} X_{0}^{*}, \quad \mathfrak{N}_{*}:=\operatorname{Ker} A_{* 0}=\operatorname{Ker} X_{0}^{*}, \quad n_{*}:=\operatorname{dim} \mathfrak{N}_{*},
$$

and require that the following condition hold true.

Condition 3.4.

$$
n \leq n_{*} \leq \infty
$$

The third condition characterizes the kernels of $X_{j}$ for $j=1, \ldots, p-1$.

Condition 3.5. The kernels of $X_{j}$ for $j=1, \ldots, p-1$ include the kernel of $X_{0}$ :

$$
\mathfrak{N} \subset \operatorname{Ker} X_{j}, \quad j=1, \ldots, p-1 .
$$

Finally, the fourth condition allows us to estimate "intermediate" operators $X_{j}$ for $j=1, \ldots, p-1$. For convenience, we also include the trivial inequality for $j=0$. 
Condition 3.6. For $j=0, \ldots, p-1$ and any $u \in \operatorname{Dom} X_{0}$, we have

$$
\left\|X_{j} u\right\|_{\mathfrak{H}_{*}} \leq \widetilde{C}\left\|X_{0} u\right\|_{\mathfrak{H}_{*}},
$$

where $\widetilde{C}$ is a constant (obviously, $\widetilde{C} \geq 1$ ).

Remark 3.7. Instead of estimate (3.2), it suffices to check the following less restrictive estimate:

$$
\left\|X_{j} u\right\|_{\mathfrak{H}_{*}} \leq \widetilde{C}_{1}\left(\left\|X_{0} u\right\|_{\mathfrak{H}_{*}}+\left\|X_{p} u\right\|_{\mathfrak{H}_{*}}\right)
$$

Estimate (3.2) follows from (3.3) if Conditions 3.2 and 3.5 are satisfied.

Proposition 3.8. Under Condition 3.6, we have

$$
\left\|X(t) u-X_{0} u\right\|_{\mathfrak{H}_{*}} \leq \widehat{C}|t|\left(\left\|X_{0} u\right\|_{\mathfrak{H}_{*}}+\|u\|_{\mathfrak{H}}\right), \quad|t| \leq 1, \quad u \in \operatorname{Dom} X_{0},
$$

where

$$
\widehat{C}=\max \left\{(p-1) \widetilde{C},\left\|X_{p}\right\|\right\} .
$$

Proof. For $u \in \operatorname{Dom} X_{0}$, we write

$$
\begin{aligned}
\| X(t) u & -X_{0} u \|_{\mathfrak{H}_{*}} \\
& =\left\|\sum_{j=1}^{p} t^{j} X_{j} u\right\|_{\mathfrak{H}_{*}} \leq \sum_{j=1}^{p}|t|^{j}\left\|X_{j} u\right\|_{\mathfrak{H}_{*}} \leq\left(\sum_{j=1}^{p-1}|t|^{j}\right) \widetilde{C}\left\|X_{0} u\right\|_{\mathfrak{H}_{*}}+|t|^{p}\left\|X_{p}\right\|\|u\|_{\mathfrak{H}},
\end{aligned}
$$

which immediately implies (3.4).

Fixing a positive number $\delta \leq \min \left(d^{0} / 36,1 / 4\right)$, we put

$$
t^{0}=t^{0}(\delta)=\min \left\{1, \frac{1}{2 \widehat{C}}, \frac{\delta^{1 / 2}}{\widehat{C}}, \frac{\delta^{1 / 2 p}}{\left\|X_{p}\right\|^{1 / p}}\right\}
$$

where $\hat{C}$ is the constant defined in (3.5). Note that, by (3.5) for $p \geq 2$, we have $\widehat{C} \geq \max \left(1,\left\|X_{p}\right\|\right)$. Therefore, the minimum in (3.6) becomes

$$
t^{0}=\frac{\delta^{1 / 2}}{\widehat{C}}
$$

Lemma 3.9. For $|t| \leq t^{0}$ and $f \in \operatorname{Dom} X_{0}$ we have

$$
\begin{aligned}
\left\|X_{0} f\right\|_{\mathfrak{H}_{*}} & \leq 2\left(\|X(t) f\|_{\mathfrak{H}_{*}}+\sqrt{\delta}\|f\|_{\mathfrak{H}}\right), \\
\|X(t) f\|_{\mathfrak{H}_{*}} & \leq \frac{3}{2}\left\|X_{0} f\right\|_{\mathfrak{H}_{*}}+\sqrt{\delta}\|f\|_{\mathfrak{H}},
\end{aligned}
$$

and (3.8) implies that

$$
\left\|X_{0} f\right\|_{\mathfrak{H}_{*}}^{2} \leq 8\left(\|X(t) f\|_{\mathfrak{H}_{*}}^{2}+\delta\|f\|_{\mathfrak{H}^{\prime}}^{2}\right)
$$

Proof. We prove (3.8). Inequality (3.9) is proved similarly. Using estimate (3.4), we get

$$
\left\|X_{0} f\right\|_{\mathfrak{H}_{*}} \leq\|X(t) f\|_{\mathfrak{H}_{*}}+\left\|\left(X(t)-X_{0}\right) f\right\|_{\mathfrak{H}_{*}} \leq\|X(t) f\|_{\mathfrak{H}_{*}}+\widehat{C}|t|\left(\left\|X_{0} f\right\|_{\mathfrak{H}_{*}}+\|f\|_{\mathfrak{H}}\right) .
$$

Hence, by (3.6),

$$
\left\|X_{0} f\right\|_{\mathfrak{H}_{*}} \leq \frac{\|X(t) f\|_{\mathfrak{H}_{*}}+\widehat{C}|t|\|f\|_{\mathfrak{H}}}{1-\widehat{C}|t|} \leq 2\left(\|X(t) f\|_{\mathfrak{H}_{*}}+\sqrt{\delta}\|f\|_{\mathfrak{H}}\right) .
$$

Let $\mathbf{F}(t, s):=\mathbb{E}_{A(t)}[0 ; s]$ denote the spectral projection of the operator $A(t)$ corresponding to the interval $[0 ; s]$, and let $\mathfrak{F}(t, s):=\mathbf{F}(t, s) \mathfrak{H}$ be the corresponding subspace. 
Proposition 3.10. For $|t| \leq t^{0}$ we have

$$
\mathbf{F}(t, \delta)=\mathbf{F}(t, 3 \delta), \quad \operatorname{rank} \mathbf{F}(t, \delta)=n .
$$

Proof. We argue by contradiction. Suppose that $\operatorname{rank} \mathbf{F}(t, \delta)<n$. Then there exists $f \in \mathfrak{N}$ with $\|f\|_{\mathfrak{H}}=1$ such that $f \perp \mathfrak{F}(t, \delta)$. In this case, $\|X(t) f\|_{\mathfrak{H}_{*}}^{2}>\delta$. But on the other hand,

$$
\|X(t) f\|_{\mathfrak{H}_{*}}^{2}=\left\|\sum_{k=0}^{p} t^{k} X_{j} f\right\|_{\mathfrak{H}_{*}}^{2}=t^{2 p}\left\|X_{p} f\right\|_{\mathfrak{H}_{*}}^{2} \leq t^{2 p}\left\|X_{p}\right\|^{2} \leq \delta,
$$

because $|t| \leq t^{0} \leq \delta^{\frac{1}{2 p}}\left\|X_{p}\right\|^{-\frac{1}{p}}$. We have used the fact that $f \in \mathfrak{N}$, whence for all $j=0, \ldots, p-1$ we have $X_{j} f=0$. This contradiction shows that $\operatorname{rank} \mathbf{F}(t, \delta) \geq n$.

Conversely, suppose that $\operatorname{rank} \mathbf{F}(t, 3 \delta)>n$. Then there exists $f \in \mathfrak{F}(t, 3 \delta)$ with $\|f\|_{\mathfrak{H}}=1$ such that $f \perp \mathfrak{N}$. Consequently, $\left\|X_{0} f\right\|_{\mathfrak{H}_{*}}^{2} \geq d^{0} \geq 36 \delta$ or $\left\|X_{0} f\right\|_{\mathfrak{H}_{*}} \geq 6 \sqrt{\delta}$. However, by (3.8),

$$
\left\|X_{0} f\right\|_{\mathfrak{H}_{*}} \leq 2\left(\|X(t) f\|_{\mathfrak{H}_{*}}+\sqrt{\delta}\|f\|_{\mathfrak{H}}\right) \leq 2(\sqrt{3}+1) \sqrt{\delta}<6 \sqrt{\delta}
$$

Again, we have a contradiction. Thus,

$$
n \leq \operatorname{rank} \mathbf{F}(t, \delta) \leq \operatorname{rank} \mathbf{F}(t, 3 \delta) \leq n,
$$

which proves (3.11).

Proposition 3.10 expresses the fact that for sufficiently small $|t|$, the spectrum of $A(t)$ does not spread much from zero and does not overlap with the rest of the spectrum. Sometimes we shall use the shorthand notation

$$
\mathbf{F}(t):=\mathbf{F}(t, \delta), \quad \mathfrak{F}(t):=\mathfrak{F}(t, \delta) .
$$

3.2. The operator $R$ and the spectral germ $S$. By $P$ and $P_{*}$ we denote the orthogonal projections of $\mathfrak{H}$ onto $\mathfrak{N}$ and of the space $\mathfrak{H}_{*}$ onto $\mathfrak{N}_{*}$, respectively. We also denote $\mathcal{D}:=\operatorname{Dom} X_{0} \cap \mathfrak{N}^{\perp}$. Since (by Condition 3.2) the point $\lambda=0$ is an isolated point of the spectrum of $A_{0}$, the sesquilinear form $\left(X_{0} \varphi, X_{0} \zeta\right)_{\mathfrak{H}_{*}}, \varphi, \zeta \in \mathcal{D}$, determines an inner product on $\mathcal{D}$, turning $\mathcal{D}$ itself into a Hilbert space. For $z \in \mathfrak{H}_{*}$ consider the equation

$$
\left(X_{0} \widehat{\varphi}, X_{0} \zeta\right)_{\mathfrak{H}_{*}}=\left(z, X_{0} \zeta\right)_{\mathfrak{H}_{*}}, \quad \zeta \in \mathcal{D},
$$

with respect to $\hat{\varphi} \in \mathcal{D}$. Since the right-hand side of (3.12) is a continuous antilinear functional of $\zeta \in \mathcal{D}$, the solution $\hat{\varphi}$ exists and is unique. In this case, $X_{0} \hat{\varphi}-z \in \mathfrak{N}_{*}$.

Now, for $\omega \in \mathfrak{N}$ we put $z=-X_{p} \omega$. We denote the corresponding solution of equation (3.12) by $\hat{\varphi}(\omega)$ and consider

$$
\Omega_{*}:=X_{0} \hat{\varphi}(\omega)+X_{p} \omega \in \mathfrak{N}_{*} .
$$

We define the operator $R$ that takes $\omega$ to $\omega_{*}$ :

$$
R: \mathfrak{N} \rightarrow \mathfrak{N}_{*}, \quad R \omega=\omega_{*} .
$$

The operator $R$ admits an alternative representation:

$$
\begin{aligned}
& R=\left.P_{*} X_{p}\right|_{\mathfrak{N}} \text { or } \\
& P_{*} X_{p} P=R \oplus \mathbb{O},
\end{aligned}
$$

where the notation on the right means that $R$ is extended by zero on $\mathfrak{N}^{\perp}$. Similarly, we construct a continuous operator $R_{*}: \mathfrak{N}_{*} \rightarrow \mathfrak{N}$. For this operator, we have

$$
R_{*}=\left.P X_{p}^{*}\right|_{\mathfrak{N}_{*}}, \quad P X_{p}^{*} P_{*}=R_{*} \oplus \mathbb{O},
$$

whence it follows that $R^{*}=R_{*}$. 
Definition 3.11. The spectral germ of the family $A(t)$ at the origin is the selfadjoint operator

$$
S:=R^{*} R: \mathfrak{N} \rightarrow \mathfrak{N} .
$$

The representations (3.15) -3.17 imply that

$$
S=\left.P X_{p}^{*} P_{*} X_{p}\right|_{\mathfrak{N}}, \quad S \oplus \mathbb{O}=P X_{p}^{*} P_{*} X_{p} P .
$$

By Condition 3.2 the operator $S$ acts in a finite-dimensional subspace.

Remark 3.12. The germ $S$ is only determined by the operators $X_{0}$ and $X_{p}$, and it does not depend on $X_{j}$ for $j=1, \ldots, p-1$.

Definition 3.13. The spectral germ $S$ is said to be nondegenerate if

$$
\operatorname{Ker} S=\{0\} \text {. }
$$

Condition (3.20) is equivalent to saying that $\operatorname{Ker} R=\{0\}$ or, equivalently, rank $R=n$.

Now, let $\widetilde{A}(t)$ be another operator family in $\mathfrak{H}$, subject to the same conditions as the family $A(t)$. The objects related to the family $\widetilde{A}(t)$ will also be marked by a tilde sign.

Definition 3.14. Families $A(t)$ and $\widetilde{A}(t)$ are threshold equivalent if $\mathfrak{N}=\tilde{\mathfrak{N}}$ and $S=\widetilde{S}$.

3.3. Eigenvalues and eigenvectors of the operator $A(t)$ in a neighborhood of zero. Consider the problem on the eigenvalues of $A(t)$ near $t=0$. In accordance with analytic perturbation theory (see $[\mathrm{K}]$ ), for $|t| \leq t^{0}$ there exist real-analytic functions $\lambda_{j}(t)$ and real-analytic $\mathfrak{H}$-valued functions $\varphi_{j}(t)$ such that

$$
A(t) \varphi_{j}(t)=\lambda_{j}(t) \varphi_{j}(t), \quad j=1, \ldots, n, \quad|t| \leq t^{0},
$$

where $t^{0}$ is the parameter defined in (3.7). The functions $\left\{\varphi_{j}(t)\right\}_{j=1}^{n}$ form an orthonormal basis in $\mathfrak{F}(t)$. The condition $|t| \leq t^{0}$ ensures that the spectral multiplicity of $A(t)$ on the interval $[0 ; \delta]$ is constant and is equal to $n$, and that there is no spectrum on $(\delta ; 3 \delta)$. For sufficiently small $t_{*} \leq t^{0}$ we have convergent power series expansions

$$
\begin{aligned}
& \lambda_{j}(t)=\gamma_{j}^{(2)} t^{2}+\gamma_{j}^{(3)} t^{3}+\cdots, \quad j=1, \ldots, n, \quad|t| \leq t_{*}, \\
& \varphi_{j}(t)=\omega_{j}+t \varphi_{j}^{(1)}+t^{2} \varphi_{j}^{(2)}+\cdots, \quad j=1, \ldots, n, \quad|t| \leq t_{*} .
\end{aligned}
$$

Theorem 3.15. The series for the eigenvalues $\lambda_{j}(t)$ begin from the power $2 p$, that is, $\gamma_{j}^{(i)}=0, i<2 p$, and

$$
\lambda_{j}(t)=\gamma_{j} t^{2 p}+\cdots, \quad \gamma_{j} \geq 0, \quad j=1, \ldots, n, \quad|t| \leq t_{*} .
$$

At the same time,

$$
S \omega_{j}=\gamma_{j} \omega_{j}, \quad j=1, \ldots, n .
$$

Proof. Condition 3.5 is crucial for the proof. The spectral problem (3.21) is equivalent to the relations

$$
\left(X(t) \varphi_{j}(t), X(t) \zeta\right)_{\mathfrak{H}_{*}}=\lambda_{j}(t)\left(\varphi_{j}(t), \zeta\right)_{\mathfrak{H}}, \quad \zeta \in \operatorname{Dom} X_{0}
$$

We substitute the power expansions (3.22) and (3.23) in (3.26) and compare the coefficients of the terms of the same order of magnitude with respect to $t$. At the zero order, we have

$$
\left(X_{0} \omega_{j}, X_{0} \zeta\right)_{\mathfrak{H}_{*}}=0, \quad \zeta \in \operatorname{Dom} X_{0},
$$

so that $\omega_{j} \in \mathfrak{N}$. Moreover, by the orthonormality of the eigenfunctions $\varphi_{j}(t)$, the set $\left\{\omega_{j}\right\}_{j=1}^{n}$ forms an orthonormal basis in $\mathfrak{N}$. Next, for the coefficients of $t^{1}$ we have

$$
\left(X_{0} \varphi_{j}^{(1)}+X_{1} \omega_{j}, X_{0} \zeta\right)_{\mathfrak{H}_{*}}+\left(X_{0} \omega_{j}, X_{1} \zeta\right)_{\mathfrak{H}_{*}}=\left(X_{0} \varphi_{j}^{(1)}, X_{0} \zeta\right)_{\mathfrak{H}_{*}}=0, \quad \zeta \in \operatorname{Dom} X_{0}
$$


because $\omega_{j} \in \mathfrak{N} \subset \operatorname{Ker} X_{1}$. Consequently, $\varphi_{j}^{(1)} \in \mathfrak{N}$. For $p>2$, the coefficients of $t^{2}$ yield

$$
\begin{array}{r}
\left(X_{0} \varphi_{j}^{(2)}+X_{1} \varphi_{j}^{(1)}+X_{2} \omega_{j}, X_{0} \zeta\right)_{\mathfrak{H}_{*}}+\left(X_{0} \varphi_{j}^{(1)}+X_{1} \omega_{j}, X_{1} \zeta\right)_{\mathfrak{H}_{*}}+\left(X_{0} \omega_{j}, X_{2} \zeta\right)_{\mathfrak{H}_{*}} \\
=\left(X_{0} \varphi_{j}^{(2)}, X_{0} \zeta\right)_{\mathfrak{H}_{*}}=\Gamma_{j}^{(2)}\left(\omega_{j}, \zeta\right)_{\mathfrak{H}}, \quad \zeta \in \operatorname{Dom} X_{0}
\end{array}
$$

For $p=2$ the argument only becomes easier, and in place of the last identity we immediately obtain (3.28). Putting $\zeta=\omega_{j}$ in (3.27), we obtain $\gamma_{j}^{(2)}=0$, whence

$$
\left(X_{0} \varphi_{j}^{(2)}, X_{0} \zeta\right)_{\mathfrak{H}_{*}}=0, \quad \zeta \in \operatorname{Dom} X_{0}
$$

Therefore, $\varphi_{j}^{(2)} \in \mathfrak{N}$. This procedure can be continued up to the power $t^{p-1}$ inclusive. As a result, we see that $\varphi_{j}^{(i)} \in \mathfrak{N}$ and $\gamma_{j}^{(i)}=0$ for $i=1, \ldots, p-1$. Now we consider the terms of degree $t^{p}$. Acting as above, we get $\gamma_{j}^{(p)}=0$. Consequently,

$$
\left(X_{0} \varphi_{j}^{(p)}+X_{p} \omega_{j}, X_{0} \zeta\right)_{\mathfrak{H}_{*}}=0, \quad \zeta \in \operatorname{Dom} X_{0},
$$

and $X_{0} \varphi_{j}^{(p)}+X_{p} \omega_{j} \in \mathfrak{N}_{*}$. By the definition of $\hat{\varphi}\left(\omega_{j}\right)$ we have $X_{0} \hat{\varphi}\left(\omega_{j}\right)+X_{p} \omega_{j} \in \mathfrak{N}_{*}$ (see (3.13)), whence

$$
X_{0}\left(\varphi_{j}^{(p)}-\widehat{\varphi}\left(\omega_{j}\right)\right) \in \mathfrak{N}_{*}
$$

We deduce that

$$
\varphi_{j}^{(p)}-\hat{\varphi}\left(\omega_{j}\right) \in \mathfrak{N},
$$

and therefore, in accordance with (3.13), (3.14),

$$
X_{0} \varphi_{j}^{(p)}+X_{p} \omega_{j}=R \omega_{j} .
$$

Consideration of the equations that correspond to $t^{i}$ for $i=p+1, \ldots, 2 p-1$ shows (via the substitution $\zeta=\omega_{j}$ ) that for these $i$ the coefficients $\gamma_{j}^{(i)}$ vanish. The equation corresponding to $t^{2 p}$ leads to

$$
\begin{aligned}
\left(X_{0} \varphi_{j}^{(p)}\right. & \left.+X_{p} \omega_{j}, X_{p} \zeta\right)_{\mathfrak{H}_{*}}+\left(X_{0} \varphi_{j}^{(p+1)}+X_{1} \varphi_{j}^{(p)}+X_{p} \varphi_{j}^{(1)}, X_{p-1} \zeta\right)_{\mathfrak{H}_{*}}+\cdots \\
& +\left(X_{0} \varphi_{j}^{(2 p)}+\cdots+X_{p} \varphi_{j}^{(p)}, X_{0} \zeta\right)_{\mathfrak{H}_{*}}=\gamma_{j}^{(2 p)}\left(\omega_{j}, \zeta\right)_{\mathfrak{H}}, \quad \zeta \in \operatorname{Dom} X_{0} .
\end{aligned}
$$

If in this equation we consider only $\zeta \in \mathfrak{N}$, it becomes

$$
\left(X_{0} \varphi_{j}^{(p)}+X_{p} \omega_{j}, X_{p} \zeta\right)_{\mathfrak{H}_{*}}=\left(R \omega_{j}, X_{p} \zeta\right)_{\mathfrak{H}_{*}}=\left(S \omega_{j}, \zeta\right)_{\mathfrak{H}}=\gamma_{j}^{(2 p)}\left(\omega_{j}, \zeta\right)_{\mathfrak{H}}, \quad \zeta \in \mathfrak{N}
$$

(we have used formulas (3.15) and (3.19)). This immediately implies the relation $S \omega_{j}=$ $\gamma_{j}^{(2 p)} \omega_{j}$. Finally,

$$
\gamma_{j}=\gamma_{j}^{(2 p)} \geq 0
$$

because the operator $A(t)$ is nonnegative for every $t$; therefore, its eigenvalues $\lambda_{j}(t)$ are nonnegative and $\gamma_{j}^{(2 p)} t^{2 p}$ is the principal part of $\lambda_{j}(t)$ in a neighborhood of $t=0$.

Relation (3.25) means that

$$
S P=\sum_{j=1}^{n} \gamma_{j}\left(\cdot, \omega_{j}\right)_{\mathfrak{H}} \omega_{j} .
$$

By (3.21) and Proposition 3.10, we have

$$
A(t) \mathbf{F}(t)=\sum_{j=1}^{n} \lambda_{j}(t)\left(\cdot, \varphi_{j}(t)\right)_{\mathfrak{H}} \varphi_{j}(t), \quad|t| \leq t^{0} .
$$


Combining this and (3.24), (3.23), (3.29), we conclude that the following power expansion is valid for sufficiently small $t_{*} \leq t^{0}$ :

$$
A(t) \mathbf{F}(t)=t^{2 p} S P+\cdots, \quad|t| \leq t_{*} .
$$

\section{§4. Abstract approach: Estimates for resolvents}

4.1. Auxiliary material. This section generalizes the arguments of Subsection 1.2 in [BS1]. For ease of reading, the main points of BS1] will also be summarized.

We are talking about estimates related to the difference of the resolvents of two operators. The standard resolvent identity is not always applicable, because the operators may have different domains. Instead, a modification of the resolvent identity is used for the case where the domains of the forms corresponding to the operators coincide.

Let $a$ and $b$ be two closed nonnegative forms in $\mathfrak{H}$ defined on a common domain

$$
\mathfrak{d}:=\operatorname{Dom} a=\operatorname{Dom} b
$$

that is dense in $\mathfrak{H}$. The forms $a$ and $b$ correspond to operators $A$ and $B$. Consider the sesquilinear form

$$
a_{\gamma}[u, v]=a[u, v]+\gamma(u, v)_{\mathfrak{H}}, \quad \gamma>0 .
$$

The corresponding quadratic form is positive. Similarly, we introduce the form $b_{\gamma}$. The linear space $\mathfrak{d}$ is a Hilbert space $\mathfrak{d}\left(a_{\gamma}\right)$ with the scalar product (4.2). The norm in $\mathfrak{d}\left(a_{\gamma}\right)$ is denoted by $\|\cdot\|_{\mathfrak{o}}$. By the coincidence (4.1) of domains, the form $b_{\gamma}$ is continuous in $\mathfrak{d}\left(a_{\gamma}\right)$, and it generates an equivalent norm. We denote

$$
\alpha^{2}=\sup _{0 \neq u \in \mathfrak{o}} \frac{a_{\gamma}[u]}{b_{\gamma}[u]} .
$$

Consider the form $\mathfrak{t}=b-a$. Obviously, it is $a_{\gamma}$-continuous, and it gives rise to the corresponding operator $T_{\gamma}$ selfadjoint in $\mathfrak{d}$ :

$$
\mathfrak{t}[u, v]=a_{\gamma}\left[T_{\gamma} u, v\right], \quad u, v \in \mathfrak{d} .
$$

We also denote

$$
\Omega_{z}(A):=I+(z+\gamma) R_{z}(A)=(A+\gamma I) R_{z}(A),
$$

where $z$ belongs to the resolvent set of $A, z \in \rho(A)$, and $R_{z}(A)$ is the resolvent of $A$ at $z$. Similar notation is introduced for $B$. Then an analog of the standard resolvent identity holds true:

$$
R_{z}(B)-R_{z}(A)=-\Omega_{z}(A) T_{\gamma} R_{z}(B), \quad z \in \rho(A) \cap \rho(B) .
$$

The definition of the norm (4.2) in the space $\mathfrak{d}$ directly implies the inequalities

$$
\begin{aligned}
\|U\|_{\mathfrak{H}} & \leq \gamma^{-1 / 2}\|u\|_{\mathfrak{d}}, \\
\left\|R_{z}(A)\right\|_{\mathfrak{H} \rightarrow \mathfrak{d}} & \leq \gamma^{-1 / 2}\left\|\Omega_{z}(A)\right\|_{\mathfrak{H} \rightarrow \mathfrak{H}}, \\
\left\|R_{z}(A)\right\|_{\mathfrak{d} \rightarrow \mathfrak{d}} & \leq \gamma^{-1}\left\|\Omega_{z}(A)\right\|_{\mathfrak{H} \rightarrow \mathfrak{H}}, \\
\left\|\Omega_{z}(A)\right\|_{\mathfrak{o} \rightarrow \mathfrak{d}} & \leq 1+|z+\gamma| \gamma^{-1}\left\|\Omega_{z}(A)\right\|_{\mathfrak{H} \rightarrow \mathfrak{H}} .
\end{aligned}
$$

In their turn, these imply estimates to be used in what follows. By $L$ with different indices we shall denote operators continuous on $\mathfrak{d}\left(a_{\gamma}\right)$.

Proposition 4.1. In the notation introduced above, we have

$$
\begin{aligned}
& \left\|L R_{z}(A)\right\|_{\mathfrak{H}} \leq \gamma^{-1}\|L\|_{\mathfrak{o}}\left\|\Omega_{z}(A)\right\|_{\mathfrak{H}}, \quad z \in \rho(A), \\
& \left\|L R_{z}(B)\right\|_{\mathfrak{H}} \leq \alpha \gamma^{-1}\|L\|_{\mathfrak{o}}\left\|\Omega_{z}(B)\right\|_{\mathfrak{H}}, \quad z \in \rho(B),
\end{aligned}
$$

where $\alpha$ is the constant defined in (4.3). 
Proposition 4.2. Let $m$ be an integer. Then

$$
\begin{aligned}
& \left\|L_{1} \Omega_{z}(A) \ldots L_{m-1} \Omega_{z}(A) L_{m} R_{z}(A)\right\|_{\mathfrak{H}} \\
& \leq \gamma^{-1}\left\|L_{1}\right\|_{\mathfrak{o}} \ldots\left\|L_{m-1}\right\|_{\mathfrak{o}}\left\|L_{m}\right\|_{\mathfrak{o}}\left\|\Omega_{z}(A)\right\|_{\mathfrak{H}}\left(1+\frac{|z+\gamma|}{\gamma}\left\|\Omega_{z}(A)\right\|_{\mathfrak{H}}\right)^{m-1}, \\
& \quad z \in \rho(A), \\
& \left\|L_{1} \Omega_{z}(A) \ldots L_{m-1} \Omega_{z}(A) L_{m} R_{z}(B)\right\|_{\mathfrak{H}} \\
& \leq \alpha \gamma^{-1}\left\|L_{1}\right\|_{\mathfrak{o}} \ldots\left\|L_{m-1}\right\|_{\mathfrak{o}}\left\|L_{m}\right\|_{\mathfrak{d}}\left\|\Omega_{z}(B)\right\|_{\mathfrak{H}}\left(1+\frac{|z+\gamma|}{\gamma}\left\|\Omega_{z}(A)\right\|_{\mathfrak{H}}\right)^{m-1}, \\
& \quad z \in \rho(A) \cap \rho(B) .
\end{aligned}
$$

Estimates (4.5) and (4.6) are proved in [BS1, Chapter 1, (2.14) and (2.15)], and estimates (4.7) and (4.8) generalize inequality (2.17) in [BS1, Chapter 1] and can easily be established by induction.

4.2. Estimates for the difference of resolvents. We return to the consideration of the polynomial pencil $A(t)$. In this section, we shall be interested in the difference of the resolvents $R_{z}(A(t))-R_{z}(A(0))$, where $z$ belongs to the contour $\Gamma_{\delta}$ surrounding equidistantly the segment $[0, \delta]$ of the real axis at a distance $\delta$. Recall that $\delta$ is a fixed number such that $\delta \leq \min \left\{d^{0} / 36,1 / 4\right\}$, where $d^{0}$ is the distance from zero to the rest of the spectrum of $A_{0}$. We also recall that the parameter $t$ is subject to the condition $|t| \leq t^{0}$, where $t^{0}$ is defined in (3.7).

For brevity, we write $R_{z}(t)$ in place of $R_{z}(A(t))$ and $\Omega_{z}(t)$ in place of $\Omega_{z}(A(t))$. Also, we omit $\mathfrak{H}$ and $\mathfrak{H}_{*}$ in the notation of norms if this cannot lead to any misunderstanding.

Now we incorporate the objects associated with a polynomial pencil $A(t)$ in the approach of Subsection 4.1. Put

$$
\gamma=\delta, \quad \mathfrak{d}=\operatorname{Dom} X_{0}, \quad a[u]=\left\|X_{0} u\right\|^{2}, \quad b[u]=\|X(t) u\|^{2} .
$$

Using inequality (3.10), for $|t| \leq t^{0}$ we find

$$
\alpha^{2}=\sup _{\mathfrak{d} \ni u \neq 0} \frac{a_{\delta}[u]}{b_{\delta}[u]}=\sup _{\mathfrak{d} \ni u \neq 0} \frac{\left\|X_{0} u\right\|^{2}+\delta\|u\|^{2}}{\|X(t) u\|^{2}+\delta\|u\|^{2}} \leq \sup _{\mathfrak{d} \ni u \neq 0} \frac{8\|X(t) u\|^{2}+9 \delta\|u\|^{2}}{\|X(t) u\|^{2}+\delta\|u\|^{2}} \leq 9 .
$$

By Proposition 3.10, for $|t| \leq t^{0}$, the distance from the contour $\Gamma_{\delta}$ to $\sigma(A(t))$ is not less than $\delta$, whence

$$
\left\|R_{z}(t)\right\|_{\mathfrak{H}} \leq \delta^{-1}, \quad z \in \Gamma_{\delta}, \quad|t| \leq t^{0} .
$$

Then for $\Omega_{z}(t)=I+(z+\delta) R_{z}(t)$ we have

$$
\left\|\Omega_{z}(t)\right\|_{\mathfrak{H}} \leq 4, \quad z \in \Gamma_{\delta}, \quad|t| \leq t^{0} .
$$

Consider the form

$$
\begin{aligned}
\mathfrak{t}[u] & =\|X(t) u\|^{2}-\left\|X_{0} u\right\|^{2} \\
& =2 \operatorname{Re}\left(\left(t X_{1}+\cdots+t^{p} X_{p}\right) u, X_{0} u\right)+\left\|\left(t X_{1}+\cdots+t^{p} X_{p}\right) u\right\|^{2} .
\end{aligned}
$$

Let $T_{\delta}=T_{\delta}(t)$ be the operator corresponding to this quadratic form in the space $\mathfrak{d}$ with the metric generated by the form $a_{\gamma}$ with $\gamma=\delta$. The operator $T_{\delta}$ admits the representation

$$
T_{\delta}(t)=\sum_{j=1}^{2 p} t^{j} T_{\delta}^{(j)}
$$


where the operators $T_{\delta}^{(j)}$ are independent of $t$. In what follows, the operators $T_{\delta}^{(j)}$ and $T_{\delta}(t)$ will play the role of the operators $L$ in Subsection 4.1. We estimate their norms in $\mathfrak{d}$.

Proposition 4.3. For $|t| \leq t^{0}$, we have

$$
\left\|T_{\delta}(t)\right\|_{\mathfrak{o}} \leq C_{\mathrm{o}}|t|
$$

where

$$
C_{\circ}=5 \hat{C} \delta^{-1 / 2}=5\left(t^{0}\right)^{-1}
$$

and $t^{0}$ is defined in (3.7).

Proof. Using (3.4), (3.9), and the fact that $\delta \leq 1$, for $u \in \operatorname{Dom} X_{0}$, we obtain

$$
\begin{aligned}
\mid\|X(t) u\|^{2} & -\left\|X_{0} u\right\|^{2}|\leq|\|X(t) u\|-\left\|X_{0} u\right\| \mid\left(\|X(t) u\|+\left\|X_{0} u\right\|\right) \\
& \leq\left\|\left(X(t)-X_{0}\right) u\right\|\left(\|X(t) u\|+\left\|X_{0} u\right\|\right) \\
& \leq \frac{5}{2} \widehat{C}|t|\left(\left\|X_{0} u\right\|+\|u\|\right)\left(\left\|X_{0} u\right\|+\sqrt{\delta}\|u\|\right) \leq 5 \widehat{C}|t| \delta^{-1 / 2}\left(\left\|X_{0} u\right\|^{2}+\delta\|u\|^{2}\right),
\end{aligned}
$$

which gives precisely (4.12).

Proposition 4.4. If $j=1, \ldots, 2 p$, then

$$
\left\|T_{\delta}^{(j)}\right\|_{\mathfrak{o}} \leq \widetilde{B}
$$

where

$$
\widetilde{B}=2 p \widetilde{C}^{2}+\left\|X_{p}\right\|^{2} \delta^{-1},
$$

and $\widetilde{C}$ is the constant occurring in (3.2).

Proof. By (3.2), for $u \in \operatorname{Dom} X_{0}, i, j=0, \ldots, p-1$, we have

$$
\begin{aligned}
\left|\left(X_{j} u, X_{i} u\right)\right| & \leq \widetilde{C}^{2}\left\|X_{0} u\right\|^{2} \leq \widetilde{C}^{2}\left(\left\|X_{0} u\right\|^{2}+\delta\|u\|^{2}\right), \\
\left|\left(X_{j} u, X_{p} u\right)\right| & \leq \widetilde{C}\left\|X_{0} u\right\|\left\|X_{p}\right\|\|u\| \leq \widetilde{C}\left\|X_{p}\right\| 2^{-1} \delta^{-1 / 2}\left(\left\|X_{0} u\right\|^{2}+\delta\|u\|^{2}\right), \\
\left\|X_{p} u\right\|^{2} & \leq\left\|X_{p}\right\|^{2} \delta^{-1}\left(\left\|X_{0} u\right\|^{2}+\delta\|u\|^{2}\right) .
\end{aligned}
$$

Therefore, the representation (4.10) implies that

$$
\begin{aligned}
\left\|T_{\delta}^{(j)}\right\|_{\mathfrak{o}} & \leq 2 p \widetilde{C}^{2}, \quad 1 \leq j \leq p-1, \\
\left\|T_{\delta}^{(j)}\right\|_{\mathfrak{o}} & \leq 2(p-1) \widetilde{C}^{2}+\widetilde{C}\left\|X_{p}\right\| \delta^{-1 / 2}, \quad p \leq j \leq 2 p-1, \\
\left\|T_{\delta}^{(2 p)}\right\|_{\mathfrak{o}} & \leq\left\|X_{p}\right\|^{2} \delta^{-1} .
\end{aligned}
$$

Now we pass directly to estimating on the norm of the difference of the resolvents. Combining estimates (4.6), (4.9), and (4.12), we obtain

$$
\left\|T_{\delta}(t) R_{z}(t)\right\|_{\mathfrak{H}} \leq 12 C_{\circ} \delta^{-1}|t|, \quad|t| \leq t^{0}, \quad z \in \Gamma_{\delta},
$$

because $\alpha \leq 3$. Thus, (4.4) and (4.9) imply the estimate

$$
\left\|R_{z}(t)-R_{z}(0)\right\|_{\mathfrak{H}} \leq 48 C_{\circ} \delta^{-1}|t|, \quad|t| \leq t^{0}, \quad z \in \Gamma_{\delta} .
$$

We shall also need a more accurate representation for the difference of resolvents. We rewrite the resolvent identity (4.4) in the form

$$
R_{z}(t)=R_{z}(0)-\Omega_{z}(0) T_{\delta}(t) R_{z}(t)
$$


and we keep substituting this equation into itself, employing the expansion of $T_{\delta}(t)$ in the form (4.11). After $2 p$ iterations (viewing (4.17) itself as the zero iteration) we obtain an expansion of the form

$$
R_{z}(t)-R_{z}(0)=t I_{1}(z)+\cdots+t^{2 p} I_{2 p}(z)+\Psi(t, z),
$$

where the operators $I_{j}(z), j=1, \ldots, 2 p$, are expressed explicitly in terms of $R_{z}(0), \Omega_{z}(0)$ and the set of $T_{\delta}^{(j)}$, and do not depend on $t$, and $\Psi(t, z)$ is the remainder of higher order in $t$.

Proposition 4.5. For the remainder in the expansion (4.18), the following estimate holds true:

$$
\|\Psi(t, z)\| \leq \widehat{B}|t|^{2 p+1}, \quad|t| \leq t^{0}, \quad z \in \Gamma_{\delta},
$$

where

$$
\widehat{B}=c_{1}(p) \delta^{-1}\left(\widetilde{B}^{2 p}+C_{\circ}^{2 p+1}\right)
$$

with constant $c_{1}(p)$ depending only on $p$.

Proof. The remainder term $\Psi(t, z)$ is equal to the sum of terms of the type

$$
\widehat{\Xi}=t^{Q} \Omega_{z}(0) T_{\delta}^{\left(q_{1}\right)} \Omega_{z}(0) T_{\delta}^{\left(q_{2}\right)} \ldots \Omega_{z}(0) T_{\delta}^{\left(q_{m}\right)} R_{z}(0),
$$

where $m \leq 2 p, Q=q_{1}+\cdots+q_{m}>2 p$, and a term in which the dependence on $t$ is not expressed explicitly:

$$
\Xi=\left(\Omega_{z}(0) T_{\delta}(t)\right)^{2 p+1} R_{z}(t) .
$$

With the help of (4.7), the product of the form (4.21) can be estimated as follows:

$$
\|\widehat{\Xi}\| \leq 3 \delta^{-1}|t|^{Q} \prod_{j=1}^{m}\left\|T_{\delta}^{\left(q_{j}\right)}\right\|_{\mathfrak{o}}\left(1+4 \frac{|z+\delta|}{\delta}\right)^{m-1} .
$$

Since $|z+\delta| \leq 3 \delta$ for $z \in \Gamma_{\delta}$, we can apply (4.14) to obtain

$$
\|\widehat{\Xi}\| \leq 3 \delta^{-1} 13^{2 p-1} \widetilde{B}^{2 p}|t|^{2 p+1} .
$$

Relations (4.8), (4.9), (4.12) and the inequality $\alpha \leq 3$ imply the following estimate on the term (4.22):

$$
\|\Xi\| \leq 12 \delta^{-1}\left\|T_{\delta}(t)\right\|_{\mathfrak{o}}^{2 p+1}\left(1+4 \frac{|z+\delta|}{\delta}\right)^{2 p} \leq 12 \cdot 13^{2 p} \delta^{-1} C_{\circ}^{2 p+1}|t|^{2 p+1} .
$$

For the spectral projection, we have the representation (see $[\mathrm{K}]$ )

$$
\mathbf{F}(t, \delta)=\frac{-1}{2 \pi i} \oint_{\Gamma_{\delta}} R_{z}(t) \mathrm{d} z,
$$

where the contour $\Gamma_{\delta}$ is oriented counterclockwise. Consequently,

$$
\Phi(t):=\mathbf{F}(t, \delta)-P=\frac{-1}{2 \pi i} \oint_{\Gamma_{\delta}}\left(R_{z}(t)-R_{z}(0)\right) \mathrm{d} z .
$$

Using (4.16) and calculating the length of the contour $\Gamma_{\delta}$ as $2 \pi \delta+2 \delta$, we get

$$
\|\Phi(t)\| \leq 48(2 \pi)^{-1}(2 \pi \delta+2 \delta) C_{\circ} \delta^{-1}|t| \leq 48\left(1+\pi^{-1}\right) C_{\circ}|t|, \quad|t| \leq t^{0} .
$$

Also, we have the representation

$$
A(t) \mathbf{F}(t, \delta)=\frac{1}{2 \pi i} \oint_{\Gamma_{\delta}} z\left(R_{z}(0)-R_{z}(t)\right) \mathrm{d} z .
$$


By (4.18),

$$
A(t) \mathbf{F}(t, \delta)=t \widehat{I}_{1}+\cdots+t^{2 p} \widehat{I}_{2 p}+\widehat{\Psi}(t)
$$

where

$$
\widehat{I}_{j}=\frac{-1}{2 \pi i} \oint_{\Gamma_{\delta}} z I_{j}(z) \mathrm{d} z, \quad \widehat{\Psi}(t)=\frac{-1}{2 \pi i} \oint_{\Gamma_{\delta}} z \Psi(t, z) \mathrm{d} z .
$$

Inequality (4.19) yields an estimate for the remainder in (4.24):

$$
\|\hat{\Psi}(t)\| \leq B_{\circ}|t|^{2 p+1}, \quad|t| \leq t^{0}
$$

where, by (4.20), the constant $B_{\circ}$ is given by the expression

$$
B_{\circ}=2 \delta^{2} \widehat{B}(\pi+1) / \pi=2\left(1+\pi^{-1}\right) c_{1}(p) \delta\left(\widetilde{B}^{2 p}+C_{\circ}^{2 p+1}\right) .
$$

Finally, the consideration of the eigenvalue problem for the operator $A(t)$ (see Subsection 3.3 and formula (3.30) shows that

$$
\widehat{I}_{1}=\cdots=\widehat{I}_{2 p-1}=\mathbb{O}, \quad \widehat{I}_{2 p}=S P,
$$

or

$$
A(t) \mathbf{F}(t, \delta)=t^{2 p} S P+\widehat{\Psi}(t)
$$

where $\widehat{\Psi}(t)$ satisfies 4.25).

Proposition 4.6. If the families $A(t)$ and $\widetilde{A}(t)$ are threshold equivalent, then

$$
\|A(t) \mathbf{F}(t, \delta)-\widetilde{A}(t) \widetilde{\mathbf{F}}(t, \delta)\| \leq\left(B_{\circ}(A)+B_{\circ}(\widetilde{A})\right)|t|^{2 p+1}, \quad|t| \leq \min \left(t^{0}, \widetilde{t^{0}}\right) .
$$

Proof. This is a direct consequence of the representation (4.27), estimate (4.25), and Definition 3.14 of threshold equivalent families.

4.3. Resolvent of $A(t)$ near zero. Now we investigate the resolvent of $A(t)$ near the edge of the spectrum; namely, we consider the operator $\left(A(t)+\varepsilon^{2 p} I\right)^{-1}$. First, we formulate another condition.

Condition 4.7. The eigenvalues $\lambda_{j}(t)$ of the operator $A(t)$ satisfy

$$
\lambda_{j}(t) \geq c_{*} t^{2 p}, \quad j=1, \ldots, n, \quad c_{*}>0, \quad|t| \leq t^{0} .
$$

Remark 4.8. For the spectral germ, Condition 4.7 implies that $S \geq c_{*} I_{\mathfrak{N}}$, ensuring nondegeneracy. In fact, estimates of type (4.28) follow from the condition of nondegeneracy of the germ, but for a smaller interval of $t$.

Proposition 4.9. Consider the operator

$$
G=G(\varepsilon, t, \delta):=\left(A(t)+\varepsilon^{2 p} I\right)^{-1} \mathbf{F}(t, \delta)-\left(t^{2 p} S P+\varepsilon^{2 p} I\right)^{-1} P .
$$

We have

$$
\varepsilon^{2 p-1}\|G\| \leq C_{1},
$$

where the constant $C_{1}$ is given by the expression

$$
C_{1}=(2 p-1)^{\frac{2 p-1}{2 p}}(2 p)^{-1} c_{*}^{-\frac{1}{2 p}}\left(96\left(1+\pi^{-1}\right) C_{\circ}+c_{*}^{-1} B_{\circ}\right) .
$$

Proof. The terms on the right in (4.29) can be rewritten as

$$
\begin{aligned}
\left(A+\varepsilon^{2 p} I\right)^{-1} \mathbf{F} & =\left(A \mathbf{F}+\varepsilon^{2 p} I\right)^{-1} \mathbf{F}=\mathbf{F}\left(A \mathbf{F}+\varepsilon^{2 p} I\right)^{-1}, \\
\left(t^{2 p} S P+\varepsilon^{2 p} I\right)^{-1} P & =\left(t^{2 p} S+\varepsilon^{2 p} I_{\mathfrak{N}}\right)^{-1} P .
\end{aligned}
$$


By (4.28), we can write

$$
\begin{aligned}
\left\|\left(A(t)+\varepsilon^{2 p} I\right)^{-1}\right\| & \leq\left(c_{*} t^{2 p}+\varepsilon^{2 p}\right)^{-1}, \quad|t| \leq t^{0}, \\
\left\|\left(t^{2 p} S+\varepsilon^{2 p} I_{\mathfrak{N}}\right)^{-1}\right\| & \leq\left(c_{*} t^{2 p}+\varepsilon^{2 p}\right)^{-1} .
\end{aligned}
$$

Note that we have the identity

$$
\begin{aligned}
\mathbf{F}\left(A \mathbf{F}+\varepsilon^{2 p} I\right)^{-1}\left(A \mathbf{F}-t^{2 p} S P\right) & \left(t^{2 p} S P+\varepsilon^{2 p} I\right)^{-1} P \\
& =\mathbf{F}\left(t^{2 p} S P+\varepsilon^{2 p} I\right)^{-1} P-\mathbf{F}\left(A \mathbf{F}+\varepsilon^{2 p} I\right)^{-1} P,
\end{aligned}
$$

from which it immediately follows that

$$
\begin{aligned}
G= & \left(A \mathbf{F}+\varepsilon^{2 p} I\right)^{-1} \mathbf{F}-\left(t^{2 p} S P+\varepsilon^{2 p} I\right)^{-1} P \\
= & \left(A \mathbf{F}+\varepsilon^{2 p} I\right)^{-1} \mathbf{F}(\mathbf{F}-P)+(\mathbf{F}-P)\left(t^{2 p} S P+\varepsilon^{2 p} I\right)^{-1} P \\
& \quad-\mathbf{F}\left(A \mathbf{F}+\varepsilon^{2 p} I\right)^{-1}\left(A \mathbf{F}-t^{2 p} S P\right)\left(t^{2 p} S P+\varepsilon^{2 p} I\right)^{-1} P .
\end{aligned}
$$

Using (4.27) and introducing $\Phi=\mathbf{F}-P$, we have

$$
G=G_{0}+G_{1}+G_{2}
$$

where

$$
\begin{aligned}
G_{0} & =-\mathbf{F}\left(A \mathbf{F}+\varepsilon^{2 p} I\right)^{-1} \widehat{\Psi}\left(t^{2 p} S P+\varepsilon^{2 p} I\right)^{-1} P, \\
G_{1} & =\left(A+\varepsilon^{2 p} I\right)^{-1} \mathbf{F} \Phi, \\
G_{2} & =\Phi\left(t^{2 p} S P+\varepsilon^{2 p} I\right)^{-1} P .
\end{aligned}
$$

Then estimates (4.32), (4.33), and (4.23) yield

$$
\left\|G_{1}\right\|+\left\|G_{2}\right\| \leq 96\left(1+\pi^{-1}\right) C_{\circ}\left(c_{*} t^{2 p}+\varepsilon^{2 p}\right)^{-1}|t|, \quad|t| \leq t^{0},
$$

and estimates (4.32), (4.33), and (4.25) imply that

$$
\left\|G_{0}\right\| \leq B_{\circ}\left(c_{*} t^{2 p}+\varepsilon^{2 p}\right)^{-2}|t|^{2 p+1} \leq c_{*}^{-1} B_{\circ}\left(c_{*} t^{2 p}+\varepsilon^{2 p}\right)^{-1}|t|, \quad|t| \leq t^{0} .
$$

Taken together, the last two inequalities show that

$$
\|G\| \leq C_{2}|t|\left(c_{*} t^{2 p}+\varepsilon^{2 p}\right)^{-1},
$$

where $C_{2}=96\left(1+\pi^{-1}\right) C_{\circ}+c_{*}^{-1} B_{\circ}$. The Young inequality gives

$$
c_{*} t^{2 p}+\varepsilon^{2 p} \geq C_{3}|t| \varepsilon^{2 p-1},
$$

where $C_{3}=2 p c_{*}^{1 / 2 p}(2 p-1)^{-\frac{2 p-1}{2 p}}$. Consequently,

$$
|T|\left(c_{*} t^{2 p}+\varepsilon^{2 p}\right)^{-1} \leq C_{3}^{-1} \varepsilon^{-2 p+1} .
$$

Estimate (4.30) follows from (4.34) and (4.35).

Proposition 4.9 and Definition 3.14 imply the following assertion.

Proposition 4.10. Suppose the families $A(t)$ and $\widetilde{A}(t)$ are threshold equivalent, and let $\delta \leq \min \left(d^{0} / 36, \widetilde{d^{0}} / 36,1 / 4\right)$. Then for the operator-valued function

$$
J=\left(A(t)+\varepsilon^{2 p} I\right)^{-1} \mathbf{F}(t, \delta)-\left(\widetilde{A}(t)+\varepsilon^{2 p} I\right)^{-1} \widetilde{\mathbf{F}}(t, \delta),
$$

we have

for $\varepsilon>0$ and $|t| \leq \min \left(t^{0}, \widetilde{t^{0}}\right)$.

$$
\varepsilon^{2 p-1}\|J\| \leq C_{1}+\widetilde{C_{1}}
$$

In accordance with Proposition 3.10 for $|t| \leq t^{0}$ the spectrum of $A(t)$ is empty in the interval $(\delta, 3 \delta)$, and therefore $\left\|\left(A(t)+\varepsilon^{2 p} I\right)^{-1} \mathbf{F}(t, \delta)^{\perp}\right\| \leq(3 \delta)^{-1}$. Hence, estimate (4.30) and Proposition 4.10 imply the following theorem. 
Theorem 4.11. Suppose that operator families $A(t)$ and $\widetilde{A}(t)$ of the type (3.1) are threshold equivalent, let $S$ be their common spectral germ (3.18), and let $P$ be the projection onto the common kernel $\mathfrak{N}=\operatorname{Ker} A(0)=\operatorname{Ker} \widetilde{A}(0)$. Also, let $\delta \leq \min \left(d^{0} / 36, \widetilde{d}^{0} / 36,1 / 4\right)$. Then

$$
\begin{array}{r}
\varepsilon^{2 p-1}\left\|\left(A(t)+\varepsilon^{2 p} I\right)^{-1}-\left(t^{2 p} S P+\varepsilon^{2 p} I\right)^{-1} P\right\|_{\mathfrak{H} \rightarrow \mathfrak{H}} \leq C_{1}+\varepsilon^{2 p-1}(3 \delta)^{-1}, \\
\varepsilon>0, \quad|t| \leq t^{0}, \\
\varepsilon^{2 p-1}\left\|\left(A(t)+\varepsilon^{2 p} I\right)^{-1}-\left(\widetilde{A}(t)+\varepsilon^{2 p} I\right)^{-1}\right\|_{\mathfrak{H} \rightarrow \mathfrak{H}} \leq C_{1}+\widetilde{C}_{1}+2 \varepsilon^{2 p-1}(3 \delta)^{-1}, \\
\varepsilon>0, \quad|t| \leq \min \left(t^{0}, \widetilde{t^{0}}\right) .
\end{array}
$$

\section{§5. Preliminary analysis of Differential operators}

We return to the analysis of differential operators. Let the assumptions of Subsection 2.2 be satisfied, and let $A$ be the operator (2.8). In this section, we factorize the operator $A$ in the form (1.1), describe the Gelfand-Bloch transformation and reduce the problem to a situation suitable for the abstract approach of $\S \$ 3$ and 4 .

5.1. Factorization of the operator $A$. By (2.3), the tensor $g$ admits the form

$$
g=\chi^{2}
$$

where the tensor $\chi \in \mathbf{B}(M)$ satisfies (2.3) (with the replacement of the constants $\nu_{1,2}$ with their square roots) and the equivalent relations of the form (2.4). The form (2.7) and the operator (2.8) can be written as

$$
\mathfrak{a}[u]=\|X u\|_{L_{2}\left(\mathbb{R}^{d} ; M\right)}^{2}, \quad A=X^{*} X,
$$

where the operator $X$ acts from $L_{2}\left(\mathbb{R}^{d}\right)$ to $L_{2}\left(\mathbb{R}^{d} ; M\right)$ on the domain

$$
\operatorname{Dom} X=H^{p}\left(\mathbb{R}^{d}\right)
$$

by the formula

$$
X u=\chi \mathbf{D}^{p} u, \quad u \in \operatorname{Dom} X .
$$

5.2. Expansion of the operator $A$ into a direct integral. The Gelfand-Bloch transformation $\mathcal{U}$ is the unitary map

$$
\mathcal{U}: L_{2}\left(\mathbb{R}^{d}\right) \rightarrow \int_{\widetilde{\Omega}}^{\oplus} L_{2}(\Omega) \mathrm{d} \mathbf{k}
$$

defined on functions of the Schwartz class $\mathcal{S}$ by the formula

$$
\begin{array}{r}
\widetilde{v}(\mathbf{k}, \mathbf{x})=(\mathcal{U} v)(\mathbf{k}, \mathbf{x})=|\widetilde{\Omega}|^{-1 / 2} \sum_{\mathbf{b} \in \Gamma} \exp (-i\langle\mathbf{k}, \mathbf{x}+\mathbf{b}\rangle) v(\mathbf{x}+\mathbf{b}), \\
v \in \mathcal{S}\left(\mathbb{R}^{d}\right), \quad \mathbf{x} \in \mathbb{R}^{d}, \quad \mathbf{k} \in \mathbb{R}^{d},
\end{array}
$$

and extended to $L_{2}$ by continuity. We consider the closed operator

$$
X(\mathbf{k}): L_{2}(\Omega) \rightarrow L_{2}(\Omega ; M), \quad \mathbf{k} \in \mathbb{R}^{d},
$$

defined on the domain

$$
\operatorname{Dom} X(\mathbf{k})=\widetilde{H}^{p}(\Omega)
$$

and acting by the formula

$$
X(\mathbf{k})=\chi(\mathbf{x})(\mathbf{D}+\mathbf{k})^{p} .
$$

Consider the quadratic form

$$
\mathfrak{a}(\mathbf{k})[u]=\|X(\mathbf{k}) u\|_{L_{2}(\Omega ; M)}^{2}, \quad u \in \operatorname{Dom} \mathfrak{a}(\mathbf{k})=\operatorname{Dom} X(\mathbf{k}), \quad \mathbf{k} \in \mathbb{R}^{d},
$$


and the corresponding selfadjoint operator

$$
A(\mathbf{k})=X(\mathbf{k})^{*} X(\mathbf{k}): L_{2}(\Omega) \rightarrow L_{2}(\Omega), \quad \mathbf{k} \in \mathbb{R}^{d},
$$

which (see (2.8)) can formally be written as

$$
A(\mathbf{k})=\left((\mathbf{D}+\mathbf{k})^{p}\right)^{*} g(\mathbf{x})(\mathbf{D}+\mathbf{k})^{p} .
$$

The Gelfand-Bloch transformation (5.1) makes it possible to perform a partial diagonalization of the operator $A$. Namely, if $v \in \operatorname{Dom} \mathfrak{a}=\operatorname{Dom} X$ and $\mathcal{U} v=\widetilde{v}$, then

$$
\widetilde{v}(\mathbf{k}, \cdot) \in \operatorname{Dom} \mathfrak{a}(\mathbf{k})=\operatorname{Dom} X(\mathbf{k}) \quad \text { for a.e. } \mathbf{k} \in \mathbb{R}^{d}
$$

and

$$
\mathfrak{a}[v]=\int_{\widetilde{\Omega}} \mathfrak{a}(\mathbf{k})[\widetilde{v}(\mathbf{k}, \cdot)] \mathrm{d} \mathbf{k} .
$$

Conversely, if relation (5.8) is satisfied for $\widetilde{v} \in \int_{\widetilde{\Omega}}^{\oplus} L_{2}(\Omega) \mathrm{d} \mathbf{k}$, and the integral on the right in (5.9) is finite, then $v=\mathcal{U}^{-1} \widetilde{v} \in$ Dom $\mathfrak{a}$ and (5.9) holds true. Relations (5.8) and (5.9) mean that under the action of the Gelfand-Bloch transformation the operator $A$ becomes the layerwise multiplication by $A(\mathbf{k})$ for $\mathbf{k} \in \widetilde{\Omega}$ :

$$
\mathcal{U} A \mathcal{U}^{-1}=\int_{\widetilde{\Omega}}^{\oplus} A(\mathbf{k}) \mathrm{d} \mathbf{k} .
$$

5.3. The study of the operators $X(\mathbf{k})$. In the operator-valued functions of the quasiimpulse $\mathbf{k}$ introduced above, we single out the dependence on the modulus of the parameter $\mathbf{k}$ :

$$
X(\mathbf{k})=X(t, \boldsymbol{\theta})=X(t \boldsymbol{\theta}), \quad \text { where } \mathbf{k}=t \boldsymbol{\theta}, \quad t=|\mathbf{k}|, \quad \boldsymbol{\theta} \in \mathbb{S}^{d-1} .
$$

The dependence on the angular parameter $\boldsymbol{\theta}$ will often be omitted in the sequel. As a function of the scalar parameter $t$, the operator $X(t)$ can be written in the form

$$
X(t)=\sum_{j=0}^{p} t^{j} X_{j}
$$

(see (5.4)), where the operators $X_{j}$ depend only on $\boldsymbol{\theta}$. The leading part of the operator (5.11) in the neighborhood of $t=0$ is

$$
X_{0}=X(0)=\chi(\mathbf{x}) \mathbf{D}^{p}, \quad \operatorname{Dom} X_{0}=\widetilde{H}^{p}(\Omega) .
$$

The operator $X_{0}$ is defined on the same domain as $X(t)$. The "intermediate" operators

$$
X_{j}=\chi(\mathbf{x}) \operatorname{Sym}\left\{\boldsymbol{\theta}^{j} \mathbf{D}^{p-j}\right\}, \quad \operatorname{Dom} X_{j}=\widetilde{H}^{p-j}(\Omega), \quad j=1, \ldots, p-1,
$$

are also unbounded and have wider domains. Here, Sym is the symbol of symmetrization over all indices. In more detail,

$$
\begin{aligned}
\left(\left(X_{j} u\right)(\mathbf{x})\right)_{s_{1}, \ldots, s_{p}} & =\sum_{1 \leq i_{1}, \ldots, i_{p} \leq d} \chi_{s_{1}, \ldots, s_{p}, i_{1}, \ldots, i_{p}}(\mathbf{x}) \sum_{\sigma \in \mathfrak{S}_{\mathfrak{p}}} \theta_{\sigma_{1}(I)} \ldots \theta_{\sigma_{j}(I)} D_{\sigma_{j+1}(I)} \ldots D_{\sigma_{p}(I)} u(\mathbf{x}),
\end{aligned}
$$

where $\mathfrak{S}_{p}$ is the set of all permutations of order $p$, and $I=\left(i_{1}, \ldots, i_{p}\right)$. Finally,

$$
X_{p}=\chi(\mathbf{x}) \boldsymbol{\theta}^{p}
$$

is a bounded operator from $L_{2}(\Omega)$ to $L_{2}(\Omega ; M)$. It is clear that

$$
\left\|X_{p}\right\|=\|\chi\|_{\infty}=\|g\|_{\infty}^{1 / 2}=\nu_{2}^{1 / 2} .
$$


All operators $X_{j}, j=0, \ldots, p$, are closed on their domains, and the corresponding graph norm is equivalent to the standard norm in $\widetilde{H}^{p-j}(\Omega)$, due to the bilateral constraints on the tensor $\chi$.

Now we study the kernels of $X_{j}$ for $j=0, \ldots, p-1$.

Proposition 5.1. We have

$$
\mathfrak{N}:=\operatorname{Ker} X_{0}=\left\{\text { const } \in L_{2}(\Omega)\right\} \subset \operatorname{Ker} X_{j}, \quad 1 \leq j \leq p-1 .
$$

Proof. Since the tensor $\chi$ is positive definite, the relation $u \in \mathfrak{N}$ is equivalent to the fact that all the derivatives of $p$ th order of $u$ are equal to zero: $\mathbf{D}^{p} u=0$. Therefore, $u$ is a polynomial of degree $p-1$. On the other hand, $u$ is a periodic function: $u \in \widetilde{H}^{p}(\Omega)$, whence $u \equiv$ const. The last inclusion is obvious.

Remark 5.2. In fact, the kernels of the operators $X_{j}, j=1, \ldots, p-1$, coincide with $\mathfrak{N}$.

Remark 5.3. If $d>1$, the kernel of the adjoint operator $X_{0}^{*}=\left(\mathbf{D}^{p}\right)^{*} \chi^{*}$ has infinite dimension, because the equation $X_{0}^{*} u=0$ imposes only one linear condition on the tensor-valued function $u \in \operatorname{Dom} X_{0}^{*}$. For $d=1$, the kernel of $X_{0}^{*}$ is one-dimensional.

For what follows, we need to estimate the intermediate operators $X_{j}, j=1, \ldots, p-1$, in terms of the operator $X_{0}$.

Proposition 5.4. For $j=1, \ldots, p-1$ and any $u \in \operatorname{Dom} X_{0}=\widetilde{H}^{p}(\Omega)$, we have

$$
\left\|X_{j} u\right\| \leq \widetilde{C}_{j} \cdot\left\|X_{0} u\right\|
$$

where

$$
\widetilde{C}_{j}=\breve{C}(d, j, p, \Gamma) \cdot\|\chi\|_{\infty} \cdot\left\|\chi^{-1}\right\|_{\infty}=\breve{C}(d, j, p, \Gamma) \nu_{1}^{-1 / 2} \nu_{2}^{1 / 2},
$$

and the constant $\check{C}(d, j, p, \Gamma)$ depends only on $d, j, p$, and $\Gamma$.

Proof. The function $u \in \widetilde{H}^{p}(\Omega)$ can be represented as a Fourier series

$$
u(\mathbf{x})=\frac{1}{|\Omega|^{1 / 2}} \sum_{\mathbf{b} \in \widetilde{\Gamma}} e^{i\langle\mathbf{b}, \mathbf{x}\rangle} u_{\mathbf{b}}, \quad \mathbf{x} \in \Omega .
$$

At the same time,

$$
\mathbf{D}^{j} u(\mathbf{x})=\frac{1}{|\Omega|^{1 / 2}} \sum_{\mathbf{b} \in \widetilde{\Gamma}} \mathbf{b}^{j} e^{i\langle\mathbf{b}, \mathbf{x}\rangle} u_{\mathbf{b}}, \quad \mathbf{x} \in \Omega .
$$

As has already been noted, $\left|\mathbf{b}^{j}\right|=|\mathbf{b}|^{j}$. We also recall that $|b| \geq 2 r_{0}$ for $0 \neq b \in \widetilde{\Gamma}$. Consequently,

$$
\begin{aligned}
\left\|\mathbf{D}^{j} u\right\|^{2}=\sum_{\mathbf{b} \in \tilde{\Gamma}}\left|\mathbf{b}^{j}\right|^{2}\left|u_{\mathbf{b}}\right|^{2} & =\sum_{\mathbf{b} \in \tilde{\Gamma}}|\mathbf{b}|^{2 j}\left|u_{\mathbf{b}}\right|^{2} \\
& \leq\left(2 r_{0}\right)^{2(j-p)} \sum_{\mathbf{b} \in \widetilde{\Gamma}}|\mathbf{b}|^{2 p}\left|u_{\mathbf{b}}\right|^{2}=\left(2 r_{0}\right)^{2(j-p)}\left\|\mathbf{D}^{p} u\right\|^{2} .
\end{aligned}
$$

The next estimate follows from the definition of the operators $X_{j}$ :

$$
\left\|X_{j} u\right\| \leq c_{2}(d, j, p)\|\chi\|_{\infty}\left\|\mathbf{D}^{p-j} u\right\|
$$

where the constant $c_{2}(d, j, p)$ depends only on $d, p$, and $j$. Summarizing the above arguments, we get the inequality

$$
\left\|X_{j} u\right\| \leq c_{2}\|\chi\|_{\infty}\left\|\mathbf{D}^{p-j} u\right\| \leq c_{2}\left(2 r_{0}\right)^{-j}\|\chi\|_{\infty}\left\|\mathbf{D}^{p} u\right\| \leq c_{2}\left(2 r_{0}\right)^{-j}\|\chi\|_{\infty}\left\|\chi^{-1}\right\|_{\infty}\left\|X_{0} u\right\|,
$$

from which we obtain (5.14) and (5.15) with $\check{C}(d, j, p, \Gamma)=c_{2}(d, j, p)\left(2 r_{0}\right)^{-j}$. 
Finally, since the embedding of $\operatorname{Dom} \mathfrak{a}(0)=\widetilde{H}^{p}(\Omega)$ in $L_{2}(\Omega)$ is compact, the operator $A_{0}:=A(0)=X_{0}^{*} X_{0}$ has discrete spectrum, and consequently, $\lambda=0$ is an isolated point of the spectrum of $A_{0}$.

Since $|\mathbf{b}| \geq 2 r_{0}$ for $0 \neq \mathbf{b} \in \widetilde{\Gamma}$, any function $u \in \widetilde{H}^{p}(\Omega)$ such that $\int_{\Omega} u \mathrm{~d} \mathbf{x}=0$ satisfies the estimate

$$
\left\|\mathbf{D}^{p} u\right\|=\sum_{\mathbf{b} \in \widetilde{\Gamma}}|\mathbf{b}|^{2 p}\left|u_{\mathbf{b}}\right|^{2} \geq\left(2 r_{0}\right)^{2 p} \sum_{0 \neq \mathbf{b} \in \widetilde{\Gamma}}\left|u_{\mathbf{b}}\right|^{2}=\left(2 r_{0}\right)^{2 p}\|u\|^{2} .
$$

Consequently,

$$
\mathfrak{a}(0)[u]=\left\|X_{0} u\right\|^{2} \geq \nu_{1}\left\|\mathbf{D}^{p} u\right\|^{2} \geq \nu_{1}\left(2 r_{0}\right)^{2 p}\|u\|^{2}, \quad u \in \widetilde{H}^{p}(\Omega), \quad \int_{\Omega} u \mathrm{~d} \mathbf{x}=0 .
$$

Therefore, the distance from zero to the rest of the spectrum of $A_{0}$ satisfies

$$
d^{0} \geq \nu_{1}\left(2 r_{0}\right)^{2 p} .
$$

\section{§6. Applichtion of the abstract approach to Differential operators}

We apply the abstract approach of $\$ ₫ 3$ and 4 to the periodic differential operators introduced in $\$ 5$

6.1. Inclusion of differential operators in the framework of $\S 3$. First, we establish a correspondence between the notation. Now

$$
\mathfrak{H}=L_{2}(\Omega), \quad \mathfrak{H}_{*}=L_{2}(\Omega ; M), \quad m=\operatorname{dim} M .
$$

The operator $A(\mathbf{k})=A(t \boldsymbol{\theta})=A(t, \boldsymbol{\theta})$ acting in $\mathfrak{H}$ plays the role of the operator $A(t)$. It is factored as $A(t)=X(t)^{*} X(t)$, where $X(t): \mathfrak{H} \rightarrow \mathfrak{H}_{*}$ is a polynomial pencil (5.11). Also,

$$
\begin{gathered}
\mathfrak{N}=\operatorname{Ker} X(0)=\{\text { const }\} \subset L_{2}(\Omega), \quad n=\operatorname{dim} \mathfrak{N}=1, \\
\mathfrak{N}_{*}=\operatorname{Ker} X(0)^{*}, \quad n_{*}=\operatorname{dim} \mathfrak{N}_{*}, \quad n_{*}=1 \text { for } d=1, \text { and } n_{*}=\infty \text { for } d \geq 2 .
\end{gathered}
$$

Conditions 3.2, 3.4, 3.5, and 3.6 were verified in $\$ 5$,

It remains to establish Condition 4.7. For this, we use variational techniques. Denote by $E_{j}(\mathbf{k}), j \in \mathbb{N}, \mathbf{k} \in \widetilde{\Omega}$, the consequent eigenvalues of $A(\mathbf{k})$ (zone functions of the operator $A$ ). Next, in the space $\mathfrak{H}=L_{2}(\Omega)$ we consider the closed quadratic form

$$
\mathfrak{a}^{00}(\mathbf{k})[v]=\int_{\Omega}\left|(\mathbf{D}+\mathbf{k})^{p} v\right|^{2} \mathrm{~d} \mathbf{x}, \quad v \in \widetilde{H}^{p}(\Omega) .
$$

The selfadjoint operator corresponding to this form is denoted by $A^{00}(\mathbf{k})$, and its eigenvalues by $E_{j}^{00}(\mathbf{k}), j \in \mathbb{N}$. Note that

$$
\mathfrak{a}^{00}(\mathbf{k})[v]=\sum_{\mathbf{b} \in \widetilde{\Gamma}}\left|(\mathbf{b}+\mathbf{k})^{p} v_{\mathbf{b}}\right|^{2},
$$

where

$$
v(\mathbf{x})=|\Omega|^{-1 / 2} \sum_{\mathbf{b} \in \widetilde{\Gamma}} v_{\mathbf{b}} \exp (i\langle\mathbf{b}, \mathbf{x}\rangle), \quad \mathbf{x} \in \Omega .
$$

Formula (6.1) shows that the eigenvalues of $A^{00}(\mathbf{k})$ are reduced to $\left|(\mathbf{b}+\mathbf{k})^{p}\right|^{2}, \mathbf{b} \in \widetilde{\Gamma}$. Consequently, the first eigenvalue is

$$
E_{1}^{00}(\mathbf{k})=|\mathbf{k}|^{2 p}, \quad \mathbf{k} \in \widetilde{\Omega}
$$

Since $X(\mathbf{k})=\chi(\mathbf{D}+\mathbf{k})^{p}$, we have

$$
\mathfrak{a}(\mathbf{k})[u]=\|X(\mathbf{k}) u\|^{2} \geq \nu_{1} \mathfrak{a}^{00}(\mathbf{k})[u],
$$


SO

$$
E_{j}(\mathbf{k}) \geq \nu_{1} E_{j}^{00}(\mathbf{k}), \quad \mathbf{k} \in \widetilde{\Omega}, \quad j \in \mathbb{N} .
$$

Finally, from the relation (6.2) and the inequality (6.3) we obtain a lower bound for the first zone function of the operator $A$ :

$$
E_{1}(\mathbf{k}) \geq c(d, p) \nu_{1}|\mathbf{k}|^{2 p}, \quad \mathbf{k} \in \widetilde{\Omega},
$$

which implies Condition 4.7

The spectral germ $S(\boldsymbol{\theta})$ of the operator family $A(\mathbf{k})=A(t \boldsymbol{\theta})=A(t, \boldsymbol{\theta})$ now depends on $\boldsymbol{\theta}$. By Remark 4.8, the germ is nondegenerate uniformly in $\boldsymbol{\theta}$, i.e., $S(\boldsymbol{\theta}) \geq c_{*} I_{\mathfrak{N}}$, where

$$
c_{*}=\nu_{1} .
$$

6.2. Construction of the spectral germ. To construct the spectral germ $S(\boldsymbol{\theta})$ of the family $A(t, \boldsymbol{\theta})$ at zero, first we construct the operator $R(\boldsymbol{\theta})$. The construction will be carried out as it was outlined in Subsection 3.2. First, consider equation (3.12). Let

$$
\mathfrak{M}=\left\{\mathbf{q} \in \mathfrak{H}_{*}, \mathbf{q}=\text { const } \in M \simeq \mathbb{C}^{m}\right\}
$$

be a subspace of tensor constants. Then, for every $\mathbf{C} \in \mathfrak{M}$ there exists a unique $v \in \widetilde{H}^{p}(\Omega)$ such that $\int_{\Omega} v \mathrm{~d} \mathbf{x}=0$ and

$$
\left(G \mathbf{D}^{p} v, \mathbf{D}^{p} w\right)_{\mathfrak{H}_{*}}=-\left(g \mathbf{C}, \mathbf{D}^{p} w\right)_{\mathfrak{H}_{*}}, \quad w \in \widetilde{H}^{p}(\Omega) .
$$

We introduce the operator $B: \mathfrak{M} \rightarrow \mathfrak{N}_{*}$ acting by the formula

$$
B \mathbf{C}=\chi\left(\mathbf{D}^{p} v+\mathbf{C}\right) \in \mathfrak{N}_{*},
$$

where $v$ is the solution of (6.6). Note that the operator $B$ does not depend on the quasimomentum $\mathbf{k}$. Also, since $\int_{\Omega} \mathbf{D}^{p} v \mathrm{~d} \mathbf{x}=0$ for any function $v \in \widetilde{H}^{p}(\Omega)$, and $\int_{\Omega} \mathbf{C} \mathrm{d} \mathbf{x}=0$ only if $\mathfrak{M} \ni \mathbf{C}=0$, we have $\operatorname{Ker} B=\{0\}$. Consequently, $\operatorname{rank} B=m$.

Now let $c \in \mathfrak{N}$ be a scalar constant. Put $\mathbf{C}=\mathbf{C}(\boldsymbol{\theta})=\boldsymbol{\theta}^{p} c \in \mathfrak{M}$. Then

$$
R(\boldsymbol{\theta}) c=B(\mathbf{C}(\boldsymbol{\theta}))=B\left(\boldsymbol{\theta}^{p} c\right) ;
$$

i.e., $R(\boldsymbol{\theta})$ is the composition of multiplication by $\boldsymbol{\theta}^{p}$ and the operator $B$, which does not depend on $\boldsymbol{\theta}$ :

$$
R(\boldsymbol{\theta}): \mathfrak{N} \rightarrow \mathfrak{N}_{*}, \quad R(\boldsymbol{\theta})=B \circ \boldsymbol{\theta}^{p} .
$$

Obviously, $\operatorname{rank} R(\boldsymbol{\theta})=1$. In what follows, we shall indicate the dependence of the operators on tensors that determine the properties of the medium (such as the tensor $g$ ). The representation (6.8) allows us to write the germ in the form

$$
S(\boldsymbol{\theta} ; g)=(R(\boldsymbol{\theta} ; g))^{*} R(\boldsymbol{\theta} ; g)=\left(\boldsymbol{\theta}^{p}\right)^{*} B^{*} B \boldsymbol{\theta}^{p}=\left(\boldsymbol{\theta}^{p}\right)^{*} g^{0} \boldsymbol{\theta}^{p},
$$

where $g^{0}=B^{*} B: \mathfrak{M} \rightarrow \mathfrak{M}$. Since $\mathfrak{M}$ can be identified with the space $M$, the operator $g^{0}$ can be interpreted as a tensor of order $2 p$ or as a bounded operator on $M$. That is, $g^{0}$ is an object of the same nature as the initial tensor $g$. The tensor $g^{0}$ is called the effective tensor for the problem in question. It is important to note that this is a constant tensor independent of $\mathbf{x}$. 
6.3. The effective tensor and effective DO. Together with the family $A(t, \boldsymbol{\theta} ; g)$, we now consider the family $A\left(t, \boldsymbol{\theta} ; g^{0}\right)$. We may assume that $g^{0}$ is factored: $g^{0}=\left(\chi^{0}\right)^{*} \chi^{0}$. Note that if $g=g^{0}$, then the solution of (6.6) is given by $v=0$ and, consequently, $\left(g^{0}\right)^{0}=g^{0}$ and

$$
S(\boldsymbol{\theta} ; g)=S\left(\boldsymbol{\theta} ; g^{0}\right)=\left(\boldsymbol{\theta}^{p}\right)^{*} g^{0} \boldsymbol{\theta}^{p}, \quad \boldsymbol{\theta} \in \mathbb{S}^{d-1} .
$$

The latter observation shows that the families $A(t, \boldsymbol{\theta} ; g)$ and $A\left(t, \boldsymbol{\theta} ; g^{0}\right)$ are threshold equivalent. Put

$$
S\left(\mathbf{k} ; g^{0}\right)=t^{2 p} S\left(\boldsymbol{\theta} ; g^{0}\right)=\left(\mathbf{k}^{p}\right)^{*} g^{0} \mathbf{k}^{p}, \quad \mathbf{k}=t \boldsymbol{\theta} \in \mathbb{R}^{d} ;
$$

this is the symbol of a differential operator with constant coefficients:

$$
A^{0}=\left(\mathbf{D}^{p}\right)^{*} g^{0} \mathbf{D}^{p} .
$$

Definition 6.1. The operator $A^{0}$ is called the effective differential operator for the operator $A$.

Proposition 6.2. The effective tensor $g^{0}$ admits the representation

$$
g^{0} \mathbf{C}=|\Omega|^{-1} \int_{\Omega} g(\mathbf{x})\left(\mathbf{D}^{p} v+\mathbf{C}\right) \mathrm{d} \mathbf{x}, \quad \mathbf{C} \in \mathfrak{M},
$$

where $v$ is the solution of equation (6.6).

Proof. For $\mathbf{C} \in \mathfrak{M}$, we rewrite (6.7) in the form

$$
\chi \mathbf{C}=B \mathbf{C}-\chi \mathbf{D}^{p} v .
$$

Note that the terms on the right-hand side are orthogonal: $B \mathbf{C} \in \mathfrak{N}_{*}=\operatorname{Ker} X_{0}^{*}$ and $\chi \mathbf{D}^{p} v \in \operatorname{Ran} X_{0}$. Consequently,

$$
\|B \mathbf{C}\|_{\mathfrak{H}_{*}}^{2}=(B \mathbf{C}, \chi \mathbf{C})_{\mathfrak{H}_{*}}=\left(\chi^{*} B \mathbf{C}, \mathbf{C}\right)_{\mathfrak{H}_{*}}=\left(g\left(\mathbf{C}+\mathbf{D}^{p} v\right), \mathbf{C}\right)_{\mathfrak{H}_{*}},
$$

whence

$$
|\Omega|\left\langle g^{0} \mathbf{C}, \mathbf{C}\right\rangle_{\mathbb{C}^{m}}=\left\langle\int_{\Omega} g(\mathbf{x})\left(\mathbf{D}^{p} v+\mathbf{C}\right) \mathrm{d} \mathbf{x}, \mathbf{C}\right\rangle_{\mathbb{C}^{m}},
$$

which proves the assertion.

Also, the Voigt-Reuss fork is valid for the effective tensor $g^{0}$. The proof is exactly the same as in Subsection 3.1.4 of the paper [BS1, and we omit it here.

Proposition 6.3 (Voigt-Reuss fork). Let

$$
\bar{g}:=\frac{1}{|\Omega|} \int_{\Omega} g(\mathbf{x}) \mathrm{d} \mathbf{x}
$$

be the arithmetic mean value of the tensor $g$ on the elementary cell (taken componentwise), and let

$$
\underline{g}:=\left(\frac{1}{|\Omega|} \int_{\Omega} g(\mathbf{x})^{-1} \mathrm{~d} \mathbf{x}\right)^{-1}
$$

be the harmonic mean value of the tensor $g$. Then the effective tensor $g^{0}$ satisfies the estimates

$$
\underline{g} \leq g^{0} \leq \bar{g}
$$


6.4. Behavior of the resolvent $\left(A+\varepsilon^{2 p} I\right)^{-1}$ as $\varepsilon \rightarrow 0$. This section is based on the results of Subsection 4.3. By inequality (6.9), we have the estimates

$$
\left\|g^{0}\right\|_{\infty} \leq\|g\|_{\infty}, \quad\left\|\left(g^{0}\right)^{-1}\right\|_{\infty} \leq\left\|g^{-1}\right\|_{\infty} .
$$

Consequently, the effective tensor $g^{0}$ also satisfies a condition of the form (2.3), and with the same constants.

In accordance with the abstract approach (see Subsection 3.1), we need to fix a positive number $\delta \leq \min \left\{d^{0} / 36,1 / 4\right\}$. Looking at (5.16), we fix $\delta$ as follows:

$$
\delta=\min \left\{\nu_{1} r_{0}^{2 p} / 36,1 / 4\right\} .
$$

Next, the constant $\widetilde{C}$ in (3.2) is given by

$$
\widetilde{C}=\max \left\{1, \widetilde{C}_{1}, \ldots, \widetilde{C}_{p-1}\right\},
$$

where the $\widetilde{C}_{j}$ are as defined in (5.15). In terms of the constants $\nu_{1}, \nu_{2}$ occurring in (2.3), and taking the obvious inequality $\nu_{1}^{-1} \nu_{2} \geq 1$ into account, we have

$$
\widetilde{C} \leq c_{3}(d, p, \Gamma) \nu_{1}^{-1 / 2} \nu_{2}^{1 / 2},
$$

where $c_{3}(d, p, \Gamma)$ is a constant depending only on $d, p$, and $\Gamma$. By (6.11) and (5.12), for the constant $\widehat{C}$ as in (3.5), we have

$$
\widehat{C} \leq \nu_{2}^{1 / 2} \max \left\{1, \check{c}_{3}(d, p, \Gamma) \nu_{1}^{-1 / 2}\right\},
$$

where $\breve{c}_{3}(d, p, \Gamma)=(p-1) c_{3}(d, p, \Gamma)$.

Given (6.10) and (6.12), instead of the sharp value of the constant $t^{0}$ defined in (3.7), as $t^{0}$ we take the following (possibly underestimated) quantity:

$$
t^{0}=\frac{\nu_{2}^{-1 / 2} \min \left\{1, \nu_{1}^{1 / 2}\left(2 r_{0}\right)^{p} / 3\right\}}{2 \max \left\{1, \check{c}_{3} \nu_{1}^{-1 / 2}\right\}} .
$$

Now Theorem 4.11 is applicable. We only need to find the value of the constant $C_{1}$ defined in (4.31). Taking (5.12), (6.10), and (6.11) into account, we replace the constant $\widetilde{B}$, defined in (4.15), with a larger constant

$$
\widetilde{B}=2 p c_{3}^{2} \nu_{1}^{-1} \nu_{2}+4 \nu_{2} \max \left\{1,9 \nu_{1}^{-1}\left(2 r_{0}\right)^{-2 p}\right\} .
$$

In accordance with (4.13), (4.26), and (4.31), we obtain

$$
\begin{aligned}
& B_{\circ}=2\left(1+\pi^{-1}\right) c_{1}(p) \delta\left(\widetilde{B}^{2 p}+5^{2 p+1}\left(t^{0}\right)^{-2 p-1}\right), \\
& C_{1}=\check{c}(p) c_{*}^{-1 / 2 p}\left(480\left(1+\pi^{-1}\right)\left(t^{0}\right)^{-1}+c_{*}^{-1} B_{\circ}\right) .
\end{aligned}
$$

By (6.5), we have $c_{*}=\nu_{1}$. Together with (6.10), (6.14), (6.15), and (6.16), this shows that the constant $C_{1}$ depends only on $d, p, \nu_{1}, \nu_{2}$ and $\Gamma$.

Theorem 4.11 immediately implies the following proposition.

Proposition 6.4. For the operator

$$
\mathcal{G}(\varepsilon, \mathbf{k})=\left(A(\mathbf{k} ; g)+\varepsilon^{2 p} I\right)^{-1}-\left(A\left(\mathbf{k} ; g^{0}\right)+\varepsilon^{2 p} I\right)^{-1}
$$

we have the estimate

$$
\varepsilon^{2 p-1}\|\mathcal{G}(\varepsilon, \mathbf{k})\|_{\mathfrak{H} \rightarrow \mathfrak{H}} \leq 2 C_{1}+2 \varepsilon^{2 p-1}(3 \delta)^{-1}, \quad \varepsilon>0, \quad|\mathbf{k}| \leq t^{0},
$$

where $\delta$ and $t^{0}$ are defined in (6.10) and (6.13), respectively. The constant $C_{1}$ is defined in (6.16) and depends only on $d, p, \nu_{1}, \nu_{2}$, and $\Gamma$.

Also, we shall need estimates for $|\mathbf{k}|>t^{0}$. 
Proposition 6.5. We have

$$
\left\|\left(A(\mathbf{k} ; g)+\varepsilon^{2 p} I\right)^{-1}\right\|_{\mathfrak{H} \rightarrow \mathfrak{H}} \leq c_{4}(d, p) \nu_{1}^{-1}\left(t^{0}\right)^{-2 p}, \quad \mathbf{k} \in \operatorname{clos} \widetilde{\Omega} \cap\left\{\mathbf{k}:|\mathbf{k}|>t^{0}\right\},
$$

and the same estimate is valid for $A\left(\mathbf{k} ; g^{0}\right)$.

Proof. This claim follows immediately from estimate (6.4) on the first eigenvalue of $A(\mathbf{k} ; g)$.

On the basis of Propositions 6.4 and 6.5 we obtain the following theorem.

Theorem 6.6. In the notation introduced above, we have the following estimate:

$$
\varepsilon^{2 p-1}\|\mathcal{G}(\varepsilon, \mathbf{k})\|_{\mathfrak{H} \rightarrow \mathfrak{H}} \leq \mathcal{C}_{\times}, \quad 0<\varepsilon \leq 1, \quad \mathbf{k} \in \operatorname{clos} \widetilde{\Omega} .
$$

The constant $\mathcal{C}_{\times}$is defined by the relation

$$
\mathcal{C}_{\times}=\max \left\{C_{1}+22(3 \delta)^{-1}, 2 c_{4}(d, p) \nu_{1}^{-1}\left(t^{0}\right)^{-2 p}\right\}
$$

and depends only on $d, p, \nu_{1}, \nu_{2}$, and $\Gamma$.

Recalling the expansion (5.10) of the operator $A$ into a direct integral, we obtain

$$
\left(A(g)+\varepsilon^{2 p} I\right)^{-1}=\mathcal{U}^{-1}\left(\int_{\widetilde{\Omega}}^{\oplus}\left(A(\mathbf{k} ; g)+\varepsilon^{2 p} I\right)^{-1} \mathrm{~d} \mathbf{k}\right) \mathcal{U} .
$$

A similar representation is true if we replace $g$ by $g^{0}$. Combining this and Theorem 6.6. we obtain one of the main results.

Theorem 6.7. Let $A(g)=\left(\mathbf{D}^{p}\right)^{*} g(\mathbf{x}) \mathbf{D}^{p}$, and let $A^{0}=A\left(g^{0}\right)=\left(\mathbf{D}^{p}\right)^{*} g^{0} \mathbf{D}^{p}$ be the effective $D O$ for $A(g)$. Then

$$
\left.\varepsilon^{2 p-1} \|\left(A(g)+\varepsilon^{2 p} I\right)^{-1}-\left(A\left(g^{0}\right)+\varepsilon^{2 p} I\right)^{-1}\right) \|_{L_{2}\left(\mathbb{R}^{d}\right) \rightarrow L_{2}\left(\mathbb{R}^{d}\right)} \leq \mathcal{C}_{\times}, \quad 0<\varepsilon \leq 1,
$$

where the constant $\mathcal{C}_{\times}$is the same as in Theorem 6.6.

6.5. Proof of Theorem 2.3. Let $T_{\varepsilon}$ denote the scaling operator

$$
\left(T_{\varepsilon} u\right)(\cdot)=\varepsilon^{d / 2} u(\varepsilon \cdot),
$$

which is unitary in $L_{2}\left(\mathbb{R}^{d}\right)$. Obviously, we have the identities

$$
\begin{aligned}
& \left(A_{\varepsilon}(g)+I\right)^{-1}=\varepsilon^{2 p} T_{\varepsilon}^{*}\left(A(g)+\varepsilon^{2 p} I\right)^{-1} T_{\varepsilon}, \\
& \left(A\left(g^{0}\right)+I\right)^{-1}=\varepsilon^{2 p} T_{\varepsilon}^{*}\left(A\left(g^{0}\right)+\varepsilon^{2 p} I\right)^{-1} T_{\varepsilon},
\end{aligned}
$$

where $A\left(g^{0}\right)$ is the effective differential operator for $A(g)$. Subtracting the second identity from the first, and using the fact that the operator $T_{\varepsilon}$ is unitary, we see that

$$
\begin{aligned}
\left\|\left(A_{\varepsilon}(g)+I\right)^{-1}-\left(A\left(g^{0}\right)+I\right)^{-1}\right\|_{L_{2}\left(\mathbb{R}^{d}\right) \rightarrow L_{2}\left(\mathbb{R}^{d}\right)} & \\
= & \varepsilon^{2 p}\left\|\left(A(g)+\varepsilon^{2 p} I\right)^{-1}-\left(A\left(g^{0}\right)+\varepsilon^{2 p} I\right)^{-1}\right\|_{L_{2}\left(\mathbb{R}^{d}\right) \rightarrow L_{2}\left(\mathbb{R}^{d}\right)} .
\end{aligned}
$$

Now Theorem 2.3 follows directly from (6.18) and (6.19).

6.6. Acknowledgments. The author is grateful to M. Sh. Birman and T. A. Suslina for suggesting the problem.

I am also thankful to N. D. Filonov under whose supervision the original version of this article was prepared as a master thesis at the Division of Mathematical Physics of the Physics Department of the St. Petersburg State University, for his permanent interest and many useful comments and clarifications.

The author thanks T. A. Suslina for her continuous attention and an enormous help in preparing the paper for release. I would like to emphasize the role of a series of lectures given by T. A. Suslina on homogenization theory for periodic differential operators. 


\section{REFERENCES}

[BaPa] N. S. Bakhvalov and G. P. Panasenko, Homogenization of processes in periodic media, Nauka, Moscow, 1984; English transl., Homogenisation: averaging processes in periodic media, Kluwer Acad. Publ. Group, Dordrecht, 1989. MR0797571 (86m:73049) MR.1112788 (92d:73002)

[BeLP] A. Bensoussan, J.-L. Lions, and G. Papanicolaou, Asymptotic analysis for periodic structures, Stud. Math. Appl., vol. 5, North-Holland Publ. Co., Amsterdam-New York, 1978. MR0503330 (82h:35001)

[BS1] M. Sh. Birman and T. A. Suslina, Second order periodic differential operators. Threshold properties and homogenization, Algebra i Analiz 15 (2003), no. 5, 1-108; English transl., St. Petersburg Math. J. 15 (2004), no. 5, 639-714. MR2068790 (2005k:47097)

[BS2] - Homogenization with corrector term for periodic elliptic differential operators, Algebra i Analiz 17 (2005), no. 6, 1-104; English transl., St. Petersburg Math. J. 17 (2006), no. 6, 897-973. MR 2202045 (2006k:35011)

[BS3] - Homogenization with corrector for periodic differential operators. Approximation of solutions in the Sobolev class $H^{1}\left(\mathbb{R}^{d}\right)$, Algebra i Analiz 18 (2006), no. 6, 1-130; English transl., St. Petersburg Math. J. 18 (2007), no. 6, 857-955. MR2307356 (2008d:35008)

[BS4] Operator error estimates in the homogenization problem for nonstationary periodic equations, Algebra i Analiz 20 (2008), no. 6, 30-107; English transl., St. Petersburg Math. J. 20 (2009), no. 6, 873-928. MR2530894 (2010f:35017)

[ZKO] V. V. Zhikov, S. M. Kozlov, and O. A. Oleı̌nik, Homogenization of differential operators, Nauka, Moscow, 1993; English transl., Homogenization of differential operators and integral functionals, Springer-Verlag, Berlin, 1994. MR 1318242 (96h:35003a); MR1329546 (96h:35003b)

[K] T. Kato, Perturbation theory for linear operators, Springer-Verlag, Berlin, 1995. MR1335452 (96a:47025)

[S] T. A. Suslina, Homogenization of a periodic parabolic Cauchy problem, Nonlinear Equations and Spectral Theory, Amer. Math. Soc. Transl. (2), vol. 220, Amer. Math. Soc., Providence, RI, 2007, pp. 201-233. MR2343612(2008k:35030)

Department of Physics, St. Petersburg State University, Peterhoff, St. Petersburg 198504, RUSSIA

Laboratory of Analysis, Geometry, and Applications, University Paris 13, Paris, France

E-mail address: nikolai.veniaminov@gmail.com

E-mail address: veniaminov@math.univ-paris13.fr

Received 28/JAN/2010

Translated by THE AUTHOR 